\title{
Surface forces and rheology of titania in the presence of dicarboxylic acids: From molecular interactions to yield stress
}

E-Jen Teh ${ }^{\dagger \neq *}$, Yee-Kwong Leong ${ }^{\ddagger}$ and Vincent S.J Craig ${ }^{\dagger}$

${ }^{\dagger}$ Department of Applied Mathematics

Research School of Physics and Engineering

Australian National University

Canberra, ACT, 2601

Australia

¥School of Mechanical and Chemical Engineering

University of Western Australia

Crawley, WA, 6009

Australia

*Corresponding authors: e-jen.teh@anu.edu.au, Vince.Craig@anu.edu.au

\begin{abstract}
The surface forces and yield stress of titania were measured in the presence of dicarboxylic acids in order to understand the molecular basis for the observed rheological response. The yield stress was measured using the static vane technique whilst the surface forces were characterised using an Atomic Force Microscope. The trans and cis isomers of butenedioic acid (fumaric and maleic acids respectively) were chosen as the relative orientation of the carboxylic groups differs substantially; thus enabling the hypothesis that an increase in adhesion leads to an increase in yield stress as a consequence of the dicarboxylic acids participating in highly directed bridging to be tested. Unlike fumaric acid, maleic acid caused a yield stress reduction in the titania suspensions. Surface force measurements found that at low $\mathrm{pH}$, fumaric and maleic acids did not induce any additional attractive force between the titania surfaces. Instead, they served as steric barriers. However, significant differences in adhesion were observed, which can be explained in terms of the configuration of the acids at the surface. The observations are consistent with highly directed bridging (or lack of it) due to the molecular architecture of the dicarboxylic acids.
\end{abstract}


Keywords: dicarboxylic acids, rheology, atomic force microscopy, bridging, titania

\section{Introduction}

The "flow" or rheological behaviour of colloidal dispersions is highly dependent on the surface forces between colloid particles. A subtle change in surface forces can give rise to a dramatic change in the rheology, which ultimately dictates the functionality of these systems. Hence, to obtain a precise control over the flow properties of colloidal systems, an in-depth knowledge of the surface forces is crucial. Surface force characterization methods can generally be grouped into two major categories: indirect techniques that probe surface forces in the bulk environment as opposed to those that directly measure the pairwise interaction between two particles or surfaces. The rheology-yield stress characterization method deals with the bulk property of the dispersion whereby the yield stress is a function of the intermolecular surface forces and the number of particle-particle contacts. Yield stress is a valuable and effective evaluation method especially in industrial processes as it characterizes the "flow" property of the dispersion at different stages of processing which in turn determines the requirements during production and transporting.

The advent of direct characterization methods such as the surface forces apparatus ${ }^{1,2}$ and the atomic force microscope (AFM) ${ }^{3,4}$ has allowed the direct measurement of particle-particle interactions between two surfaces on a nanoscopic level in various environments. Here we seek to use measurements of surface forces to improve our understanding of the yield stress. This implies that the yield stress of a network of particles can at least be in part understood through the lens of the interactions between pairs of particles. At the most basic level, the yield stress can be seen as the incipient fracturing of the particle network, which occurs when particles begin moving relative to each other. Prior to the particles separating (or overcoming their adhesion altogether) they will, in most cases, first roll across each other as the forces rarely act perpendicular to the surfaces. If we picture this as a sphere rolling on a flat surface or two spheres rolling relative to each other as depicted in Figure 1, the surfaces are approaching at the front of the sphere whilst the surfaces are separating at the end of the sphere. If the forces measured on approach and separation are identical, (i.e. there is no hysteresis), then the energy required to push the surfaces together at the front will be recovered as the surfaces separate at the rear and no work is required. On the contrary, hysteresis between the approach and retract curves implies loss of energy and work is required. The larger the hysteresis the 
greater the work. We would therefore expect that in the former the yield stress will be very low and in the latter significant. Thus we infer on a basic level that the yield stress is directly related to the hysteresis in the force between pairs of particles measured on approach and separation for an identical network structure.

a)

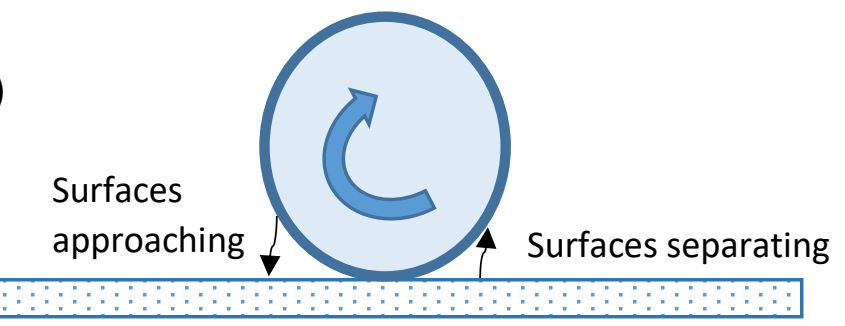

b)

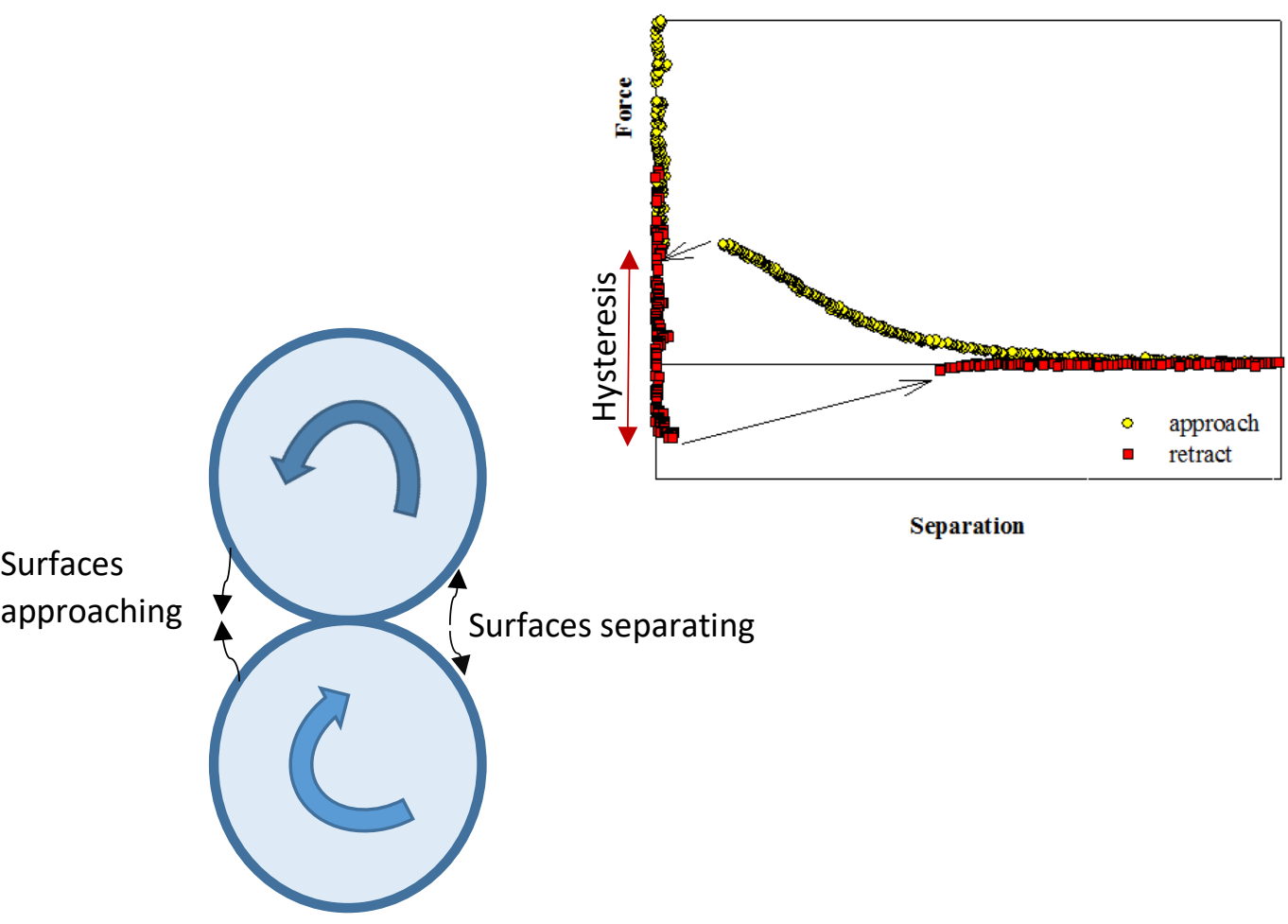

Figure 1 Schematic representation of (a) a sphere rolling on a flat surface (b) two spheres rolling relative to each other. The surfaces are approaching at the front of the sphere whilst the surfaces are separating at the end of the sphere. If the forces measured during approach and separation of the surfaces are identical, (i.e. there is no hysteresis), then the energy required to push the surfaces together at the front will be recovered as the surfaces separate at the rear and no work is required. On the contrary, hysteresis between the approach and separation curves implies loss of energy and work is required. The larger the hysteresis the greater the work.

The large yield stress increase in metal oxide dispersions upon the introduction of low molecular weight (LMW) dicarboxylic acids ${ }^{5,6,7,8}$ is often attributed to highly directed bridging. This is where 
electrostatic attraction between charged functional groups of the acids and the protonated oxide surface group on the colloids forms molecular links between particles which results in the formation of "bridges" in the network structure and thus gives rise to an increase in the interparticle strength. Despite being used on multiple occasions $s^{6,7,8,9}$ to explain the large increases in the yield stress of metal oxide dispersions, highly directed bridging has not been directly measured, unlike bridging by long chain polyelectrolytes such as PAA ${ }^{10,11}$ and polyethyleneimine (PEI) ${ }^{12}$. Additionally, the effective range of highly directed bridging and its force-distance dependency have yet to be investigated.

LMW dicarboxylic acid, ethylene dicarboxylic acid or butenedioic acid has two isomers: trans-1,2ethylene (fumaric acid) and cis-1,2-ethylene dicarboxylic acid (maleic acid). The molecular structure ensures that the carboxylic groups are oriented very differently in the two molecules. The $\mathrm{pK}_{\mathrm{a}}$ values are also significantly different whereby fumaric acid has a bulk $\mathrm{pKa} \mathrm{a}_{1}$ of 3.03 and a $\mathrm{pKa} \mathrm{a}_{2}$ of 4.54 while maleic acid has a bulk $\mathrm{pKa} \mathrm{a}_{1}$ of 1.83 and a $\mathrm{pKa}_{2}$ of $6.07^{6}$. Figure 2 shows the molecular structure of fumaric and maleic acids modelled using ChemOffice Ultra 10 . The projected size of fumaric and maleic acid molecules is approximated to be $5 \AA$ and $3 \AA$ a respectively ${ }^{6}$. Despite their small size and simple structure, both fumaric and maleic acids have been shown to change the rheology of alumina dispersions considerably. Leong ${ }^{6}$ studied the effect of molecular configuration of adsorbed fumaric and maleic acids on the rheology of $\alpha-\mathrm{Al}_{2} \mathrm{O}_{3}$ dispersions using the yield stress-vane technique and found that whilst maleic acid weakened the flocculated structure of the $\alpha-\mathrm{Al}_{2} \mathrm{O}_{3}$ dispersion, fumaric acid enhanced the strength of the flocculated structure.
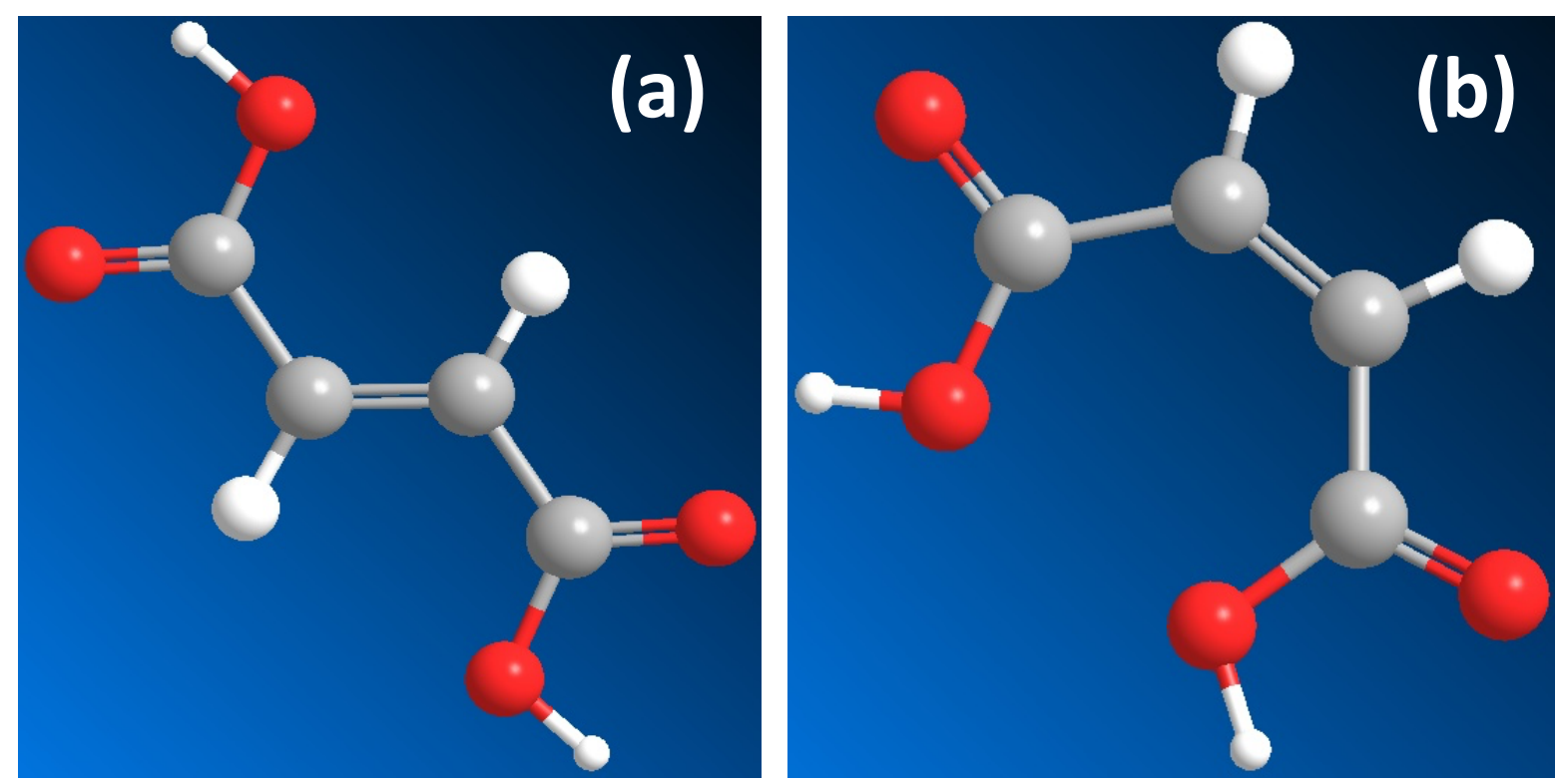

Figure 2 Molecular configuration of (a) trans-1,2-ethylene (fumaric acid) and (b) cis-1,2-ethylene dicarboxylic acid (maleic acid). Fumaric acid has a bulk pKa of 3.03 and a pKa 2 of 4.54 while maleic acid has a bulk pKa1 of 
1.83 and a $\mathrm{Ka}_{2}$ of $6.07^{6}$. Note that the red, grey and white balls represent the oxygen, carbon and hydrogen atoms respectively.

Employing smooth surfaces in AFM measurements is of utmost importance especially when short range interactions are important. Here we use Atomic Layer Deposition (ALD) to produce very smooth titanium dioxide (titania) surfaces suitable for AFM measurements. Titania was chosen as the surface charging properties of titania allow the $\mathrm{pH}$ to be varied such that the acids and surfaces can be oppositely charged over a selected pH range. Equally important, titania can be deemed as a model system as it does not undergo drastic dissolution under aqueous conditions. Additionally, titania is an important industrial commodity where it is colloidally prepared to be used in various applications such as sunscreen and paints. Our aim is to directly measure the interparticle interactions between titania surfaces in the presence of LMW dicarboxylic acids previously reported to participate in highly directed bridging. The AFM data will be compared to rheology measurements in an attempt to link the microscopic interactions and macroscopic behaviour. In particular, we will examine the surface forces to test the hypothesis that LMW dicarboxylic acids (either maleic or fumaric acid) form bridges between particles leading to an increased yield stress.

\section{Materials and Methods}

\section{Atomic Layer Deposition of Titania Surfaces}

Thin films ( $~ 5 \mathrm{~nm}$ ) of titania were grown using a Savannah 100 system with titanium isopropoxide and water as precursors as described previously ${ }^{13,14}$. The titanium isopropoxide was heated to $80^{\circ} \mathrm{C}$ during the reaction while water was at room temperature. The chamber was purged, between precursor pulses using pure nitrogen gas, for a period of $10 \mathrm{~s}$. The temperature of the chamber was maintained at $250^{\circ} \mathrm{C}$. The roughness of the surfaces after deposition of $5 \mathrm{~nm}$ of titania was measured by AFM to be $<0.5 \mathrm{~nm}$ rms over a $1 \times 1 \mu \mathrm{m}$ scan, which is similar to the underlying silicon wafer.

\section{Reagents}

The chemicals used (Sigma Aldrich) were of analytical grade and were used as received with the exception of sodium chloride $(\mathrm{NaCl})$ which was roasted at $400^{\circ} \mathrm{C}$ in an oven for 6 hours to remove any organic contaminants. 


\section{AFM experiments}

All glassware used in the AFM experiments was cleaned by soaking in $10 \mathrm{wt} \% \mathrm{NaOH}$ solution for 10 minutes prior to rinsing with copious amount of Milli-Q water (conductivity $18.5 \mathrm{M} \Omega$ ). The fluid cell, O-ring, glass syringe, Teflon tubing and tweezers were rinsed with distilled ethanol and blown dry with clean nitrogen gas. Immediately prior to the actual measurement, all the substrates, cantilevers and colloid probes were cleaned with an RF water plasma system (10 W, $60 \mathrm{~s}, 125 \mathrm{kHz}, \sim 0.1$ torr) to remove any trace of organic contaminants and to hydroxylate the surfaces. All solutions for the AFM experiments were prepared using Milli-Q water. For AFM experiments, the dicarboxylic acid concentration is given as the number of moles per litre (M). The same set of AFM solutions were subsequently used for optical reflectometry experiments.

A Digital Instruments Multimode Nanoscope III AFM fitted with a low noise head was used to measure the interaction forces between a colloid probe and substrate. Rectangular, contact mode, silicon nitride cantilevers, CSG-11 (NT-MDT) were used for all force measurements in this study. The cantilever spring constant was determined through the thermal tune method available in the Asylum picoforce AFM software. Each cantilever was calibrated prior to the attachment of a colloidal particle using the method developed by Ducker et $\mathrm{al}^{15}$ and Butt et $\mathrm{a} \mathrm{l}^{16}$. The quartz fluid cell used in the AFM experiments was sealed with a silicone O-ring. Fluids were exchanged using a clean glass syringe and Teflon tubes. The system was left to equilibrate for at least 30 minutes or until the thermal drift in the instrument had ceased before any measurements were made.

\section{Optical Reflectometry}

The optical reflectometry (OR) technique similar to that used by Djit et al. ${ }^{17}$ was employed to measure the amount of material adsorbed at the titania interfaces. A silicon wafer with $319 \pm 2 \mathrm{~nm}$ of silicon oxide and $5 \mathrm{~nm}$ of titania deposited using the ALD process was used as a substrate (see ${ }^{18}$ for details). The adsorption of molecules onto the substrate alters the ratio of the $p$ and $s$ components of the reflected light. Consequently, the change in $p / s$ ratio can be converted to surface excess if the extent of the change in the $p / s$ ratio due to a change in surface excess is known. This value, termed as the sensitivity factor, was calculated using a multi-layer Fresnel optical model ${ }^{18}$. A necessary input is the refractive index increment $(d n / d c)$ of the adsorbing species in solution. The $d n / d c$ was determined by measuring the differential refractive indices between the baseline solution and that in the presence of increasing concentration of dicarboxylic acid using a differential refractometer (Wyatt OptilabTREX). 
The experiment started with the measurement of a baseline using $\mathrm{NaCl}$ solution with no dicarboxylic acid present. Once a stable flat baseline had been obtained for some period, the experimental solution containing the dicarboxylic acid (prepared using the $\mathrm{NaCl}$ base solution) was introduced into the cell, the adsorption measured and then the solution with no dicarboxylic acid reintroduced. This cycle was repeated 3 times at each $\mathrm{pH}$ or concentration. The $\mathrm{OR}$ experiment was conducted in a temperature controlled room in which the experimental solutions were kept at least overnight prior to being used for measurements to ensure temperature equilibration. The surface excess measured by the $O R$ technique is accurate to $0.02 \mathrm{mg} / \mathrm{m}^{218}$.

\section{Titania particles}

The titania $\left(\mathrm{TiO}_{2}\right)$ particles used in this study, commercially named SG TO200, were purchased from Sukgyung Korea. The anatase titania nanoparticles are of high purity and according to the manufacturer, have an average size of $0.718 \mu \mathrm{m}$ and a BET surface area of $20.8 \mathrm{~m}^{2} \mathrm{~g}^{-1}$. Figure 3 shows an SEM image of the SG TO200 titania powder. The titania particles were moderately aspherical. The bare titania surfaces were naturally rough exhibiting deep surface crevices. Note that the thin layer of conductive platinum coating applied to reduce charging during imaging can exacerbate the appearance of surface roughness.

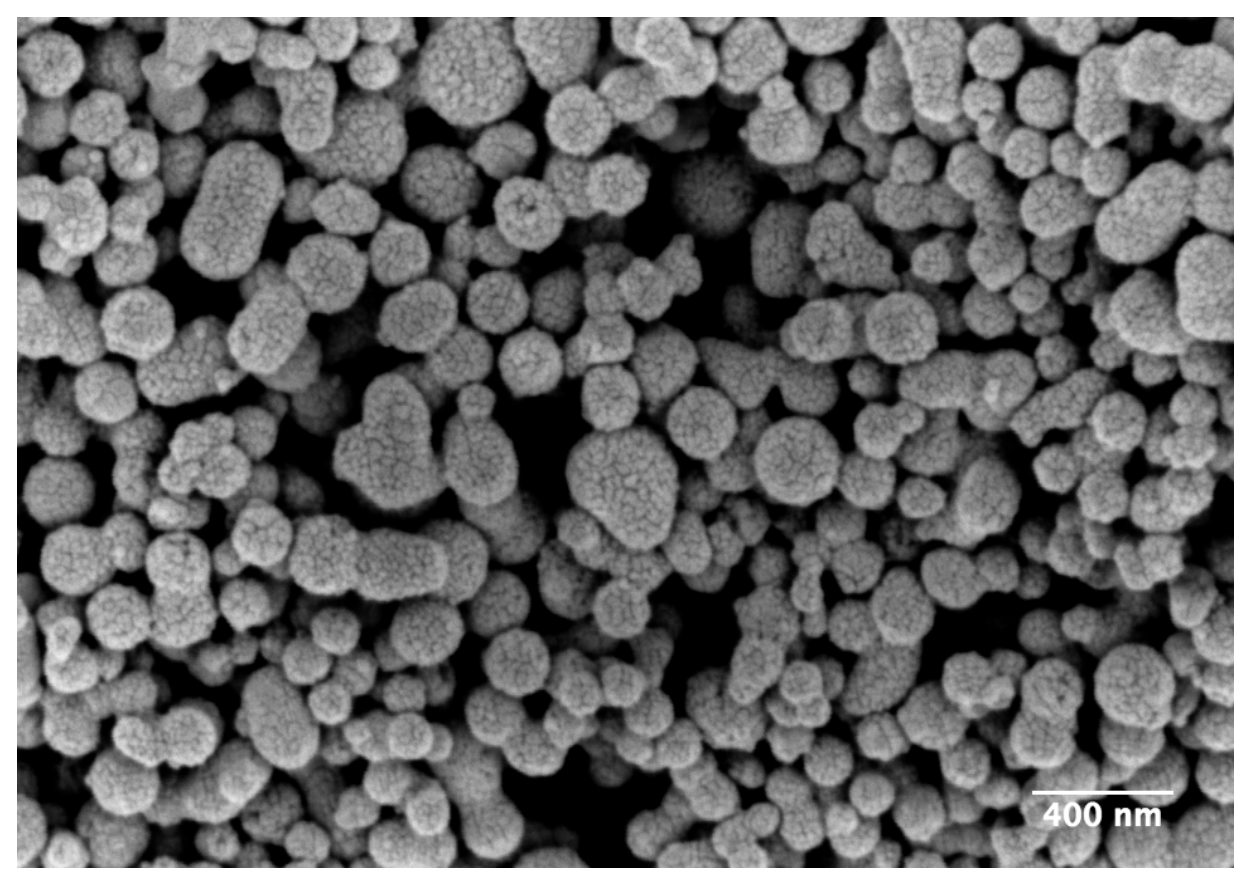

Figure 3 Scanning Electron Microscope image of SG TO200 titania particles with an average size of $0.718 \mu \mathrm{m}$ and a BET surface area of $20.8 \mathrm{~m}^{2} \mathrm{~g}^{-1}$. 


\section{Yield Stress}

The maximum torque of the slurry was measured using a Brookfield DVII+ viscometer via the vane technique ${ }^{19}$ and then converted to the corresponding yield stress. Immediately prior to the yield stress measurement, the dispersion was stirred manually with a spatula. The vane was then gently immersed into the dispersion of interest and the yield stress measured 3 times at each $\mathrm{pH}$. During the stepwise $\mathrm{pH}$ adjustment, the slurry was stirred manually with a metal spatula to promote suspension homogeneity and to decrease any localized flocculation. The $\mathrm{pH}$ of the dispersion was measured before and after the yield stress measurement to ensure its stability. The yield stress values obtained in this study are repeatable and estimated to be accurate to $15 \%$.

All dispersions used in the zeta potential and rheology studies were prepared via ultrasonification using a Branson digital Sonifier ( $20 \mathrm{kHz}, 600 \mathrm{~W}$ ). The zeta potential of the suspensions was measured using the electroacoustic method via a ZetaProbe instrument (Colloidal Dynamics, USA). The metal oxide suspensions for zeta potential measurements had an initial solid loading of $1.48 \mathrm{vol} \%$ (5.2 wt\% solid). Titania slurries for rheology measurements were prepared in batches of $\sim 80 \mathrm{~g}$. An appropriate amount of dicarboxylic acid was dissolved in purified water (conductivity $<1 \mu \mathrm{S} / \mathrm{cm}$ ) before addition to the metal oxide particles to achieve the solid loading desired. The $\mathrm{pH}$ was then adjusted to $\sim \mathrm{pH} 12$ using $5 \mathrm{M} \mathrm{NaOH}$ prior to measurement. Concentrated $\mathrm{HCl}$ was used to decrease the $\mathrm{pH}$ stepwise to 2 to minimize dilution. An Orion 3 Star Benchtop $\mathrm{pH}$ meter was used to measure the $\mathrm{pH}$ while a TPS WP-81 pH-conductivity-salinity meter was used to determine the conductivity of the slurries. Prior to each experiment, the $\mathrm{pH}$ meter was calibrated using buffer solution of $\mathrm{pH} 4,7$ and 10 while the conductivity probe was calibrated with $0.02 \mathrm{M} \mathrm{KCl}$ with a known conductivity of $2.76 \mathrm{mS} / \mathrm{cm}$. For yield stress measurements, the titania slurries were $55 \mathrm{wt} \%$ solid and were left overnight in the dark to allow for equilibration prior to any characterization. In the zeta potential and rheology experiments, the dicarboxylic acid concentration refers to the dry weight of the dicarboxylic acid added per gram of titanium oxide particles and is quoted in percentage (wt\%).

\section{Total Organic Carbon}

The adsorption of dicarboxylic acids to titania was investigated using the total organic carbon (TOC) method. The metal oxide dispersion of $20 \mathrm{wt} \%$ solid loading (without any dicarboxylic acids) was prepared using the same method employed to prepare suspensions for yield stress and zeta potential measurements. Subsequently, $0.5 \mathrm{wt} \%$ of the dicarboxylic acids were added to the dispersion. The titania dispersions were then left for 48 hours in the dark to allow sedimentation to occur. A sample of the supernatant liquid was collected using a syringe, filtered through a syringe membrane 
microfilter and sent for analysis. The TOC was determined using a Shimadzu combustion analyser and the amount of dicarboxylic acid adsorbed determined from a material balance.

\section{Zeta Potential}

The zeta potential of the colloidal particles was measured using a ZetaProbe (Colloidal Dynamics, USA). The ZetaProbe uses the ESA technique to measure the zeta potential of colloidal dispersions. During auto-titration, the $\mathrm{pH}$ was adjusted using $0.05 \mathrm{M} \mathrm{NaOH}$ or $0.05 \mathrm{M} \mathrm{HCl}$. The zeta potential measurements were conducted from high $(\sim \mathrm{pH} 12)$ to low $\mathrm{pH}(\sim \mathrm{pH} 2)$. In order to verify the cleanliness of the SG TO200 titania, the particles were washed using $1 \mathrm{M} \mathrm{NaOH}$ and rinsed with copious amounts of distilled water. This cleaning process did not result in a change in zeta potential.

\section{Results}

\section{Adsorption of Fumaric and Maleic Acids to Titania}

In order to understand the surface forces and the rheology of titania in the presence of dicarboxylic acids, it is necessary to quantify the adsorption or surface coverage of the acids on the titania surfaces. As the titania surfaces used in the rheology and surface force studies differ, the adsorption to both surfaces were investigated independently.

\section{ALD titania}

Optical reflectometry (OR) was used to measure the adsorption of fumaric and maleic acids to ALD titania surfaces identical to those used in the surface force studies. Typical OR data is presented in Figure 4. This was measured in $10^{-3} \mathrm{M} \mathrm{NaCl}$ at pH 2.4 whilst the presence of $0.03 \mathrm{M}$ fumaric acid was thrice cycled in and out. The average surface excess across three cycles was $0.43 \mathrm{mg} \mathrm{m}^{-2}$. The same approach was employed to obtain the surface excess at other concentrations (see Table 1). The corresponding surface coverages are also shown in Table $1^{6}$. Both fumaric and maleic acids exhibited similar surface excess at a given $\mathrm{pH}$ and concentration. 


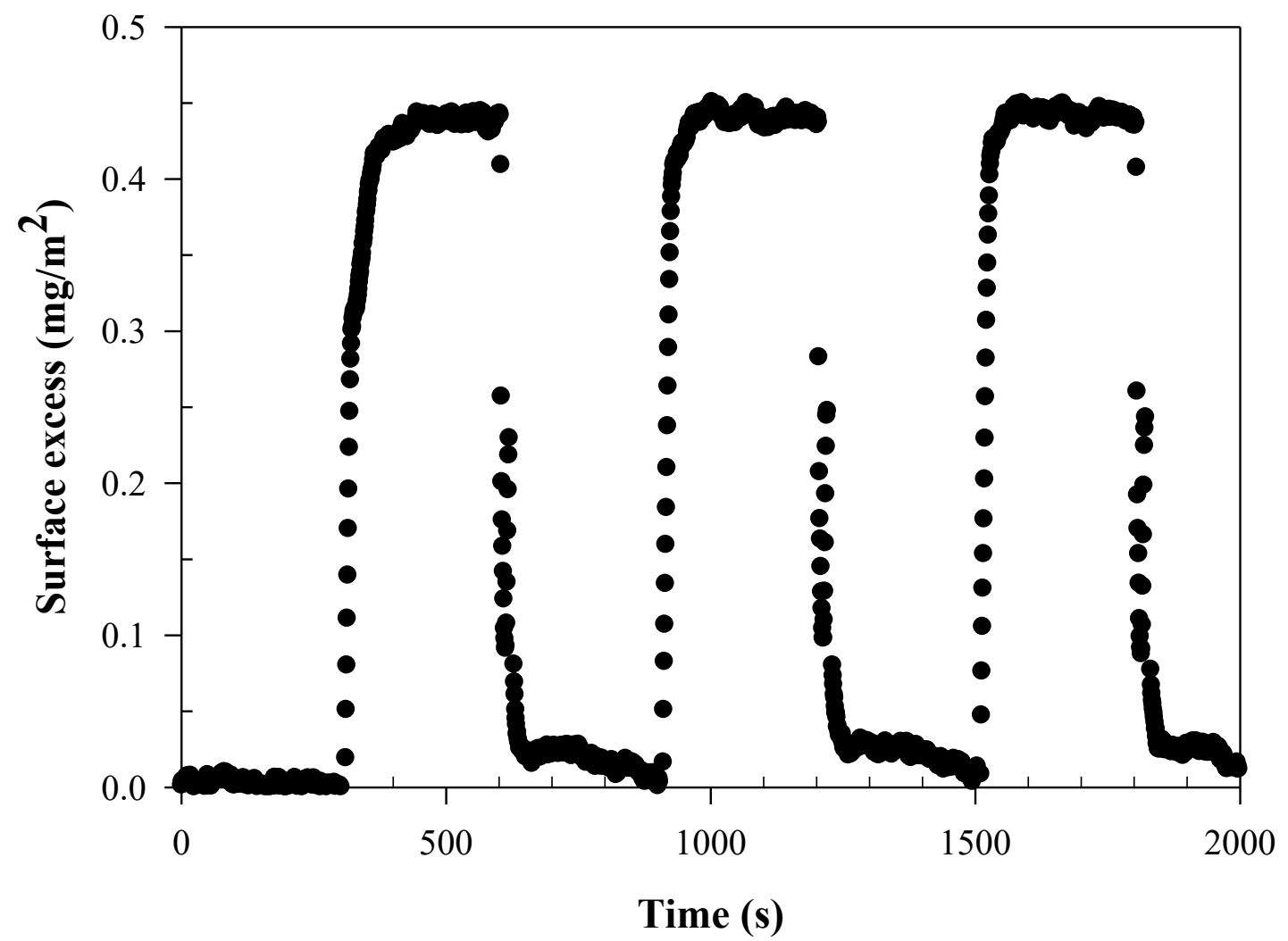

Figure 4 Surface excess as a function of time measured in $10^{-3} \mathrm{M} \mathrm{NaCl}$ at $\mathrm{pH} 2.4$ as the presence of fumaric acid was cycled in and out. Initially a stable baseline was obtained before the fumaric acid solution was introduced into the cell at approximately $300 \mathrm{~s}$ leading to a plateau in the adsorption of $0.43 \mathrm{mg} \mathrm{m}^{-2}$ of fumaric acid. At $600 \mathrm{~s}$ aqueous $10^{-3} \mathrm{M} \mathrm{NaCl}$ at $\mathrm{pH} 2.4$ is introduced into the cell causing the fumaric acid to desorb and the surface excess to return to baseline levels after $\sim 900 \mathrm{~s}$.

Two further cycles are shown with the fumaric acid being introduced at $900 \mathrm{~s}$ before being washed off and then again at $1500 \mathrm{~s}$.

Table 1 Adsorption of fumaric and maleic acids on ALD titania surfaces at $\mathrm{pH} \sim 2.5$..

\begin{tabular}{|c|c|c|c|}
\hline Acid & $\begin{array}{l}\text { Concentration } \\
\text { (M) }\end{array}$ & $\begin{array}{l}\text { Surface excess } \\
\qquad\left(\mathrm{mg} \mathrm{m}^{-2}\right)\end{array}$ & $\begin{array}{c}\text { Surface coverage* } \\
\text { (\%) }\end{array}$ \\
\hline \multirow[t]{3}{*}{ Fumaric } & $\leq 0.001$ & Negligible & 0 \\
\hline & 0.01 & 0.19 & 25 \\
\hline & 0.03 & 0.43 & 56 \\
\hline \multirow[t]{3}{*}{ Maleic } & $\leq 0.001$ & Negligible & 0 \\
\hline & 0.01 & 0.15 & 19 \\
\hline & 0.03 & 0.42 & 54 \\
\hline
\end{tabular}

*The surface coverage is based on the assumption of monolayer coverage of $0.25 \mathrm{~nm} 2$ per carboxylate. ${ }^{6}$ 


\section{SGTO200 Titania Particles}

The adsorption of fumaric and maleic acids to SGT 0200 titania particles used in the rheology studies was investigated and is presented in Figure 5 as both the amount adsorbed (left $y$-axis) and the surface coverage expressed in percentage (right $\mathrm{y}$-axis) as a function of $\mathrm{pH}$. At $\mathrm{pH}$ values above 8 , the adsorption of both acids is insignificant, this is attributed to the electrostatic repulsion arising from the negatively charged acid species and the negatively charged titania surfaces. To investigate this further, the zeta potential was measured as a function of $\mathrm{pH}$ and is shown in Figure 6 .

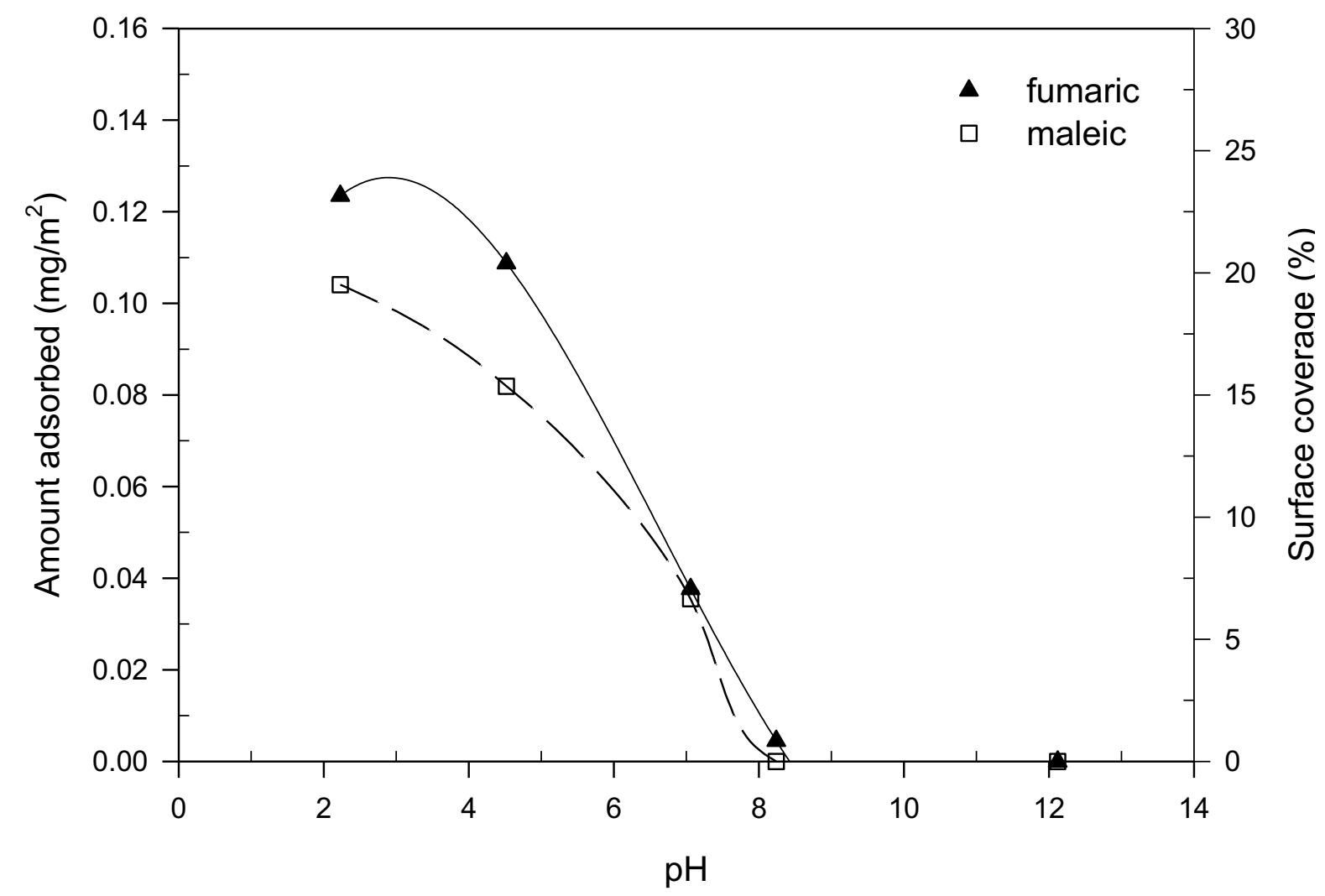

Figure 5 Adsorption of $0.5 \mathrm{wt} \%$ fumaric and maleic acids in $20 \mathrm{wt} \%$ solid SGT O200 titania suspensions expressed as amount of acid adsorbed and as surface coverage. The lines are to guide the eye.

\section{Zeta Potential of SG TO200 Titania Particles}

The isoelectric point of the SG TO200 titania particles, IEPSG TO200, is pH 6.4 (refer Figure 6). This is within the range of IEP reported for anatase particles in previous studies $20,21,22,23,24,25,26,27$. At $\mathrm{pH}$ values above the IEPSG TO200 fumaric and maleic acids had little influence on the zeta potential. Minimal changes to the zeta potential above the IEP is consistent with the adsorption studies which show that the adsorption is also negligible in this $\mathrm{pH}$ range. Below the IEP, the zeta potential is reduced in the 
presence of the dicarboxylic acids due to the adsorption of the acids to the positively charged titania surface. The adsorption studies show that there was a degree of adsorption of both fumaric and maleic acids up to pH 8 (above the IEP SG TO200). This is the origin of the shift in the IEP in the presence of these dicarboxylic acids and suggests that the titania surface has a variety of ionizing groups. Note that the ionic strength of the titania dispersions used in this study is expressed in terms of a 1:1 electrolyte and for the zeta potential measurements, the dispersion was approximately $0.02 \mathrm{M}$.

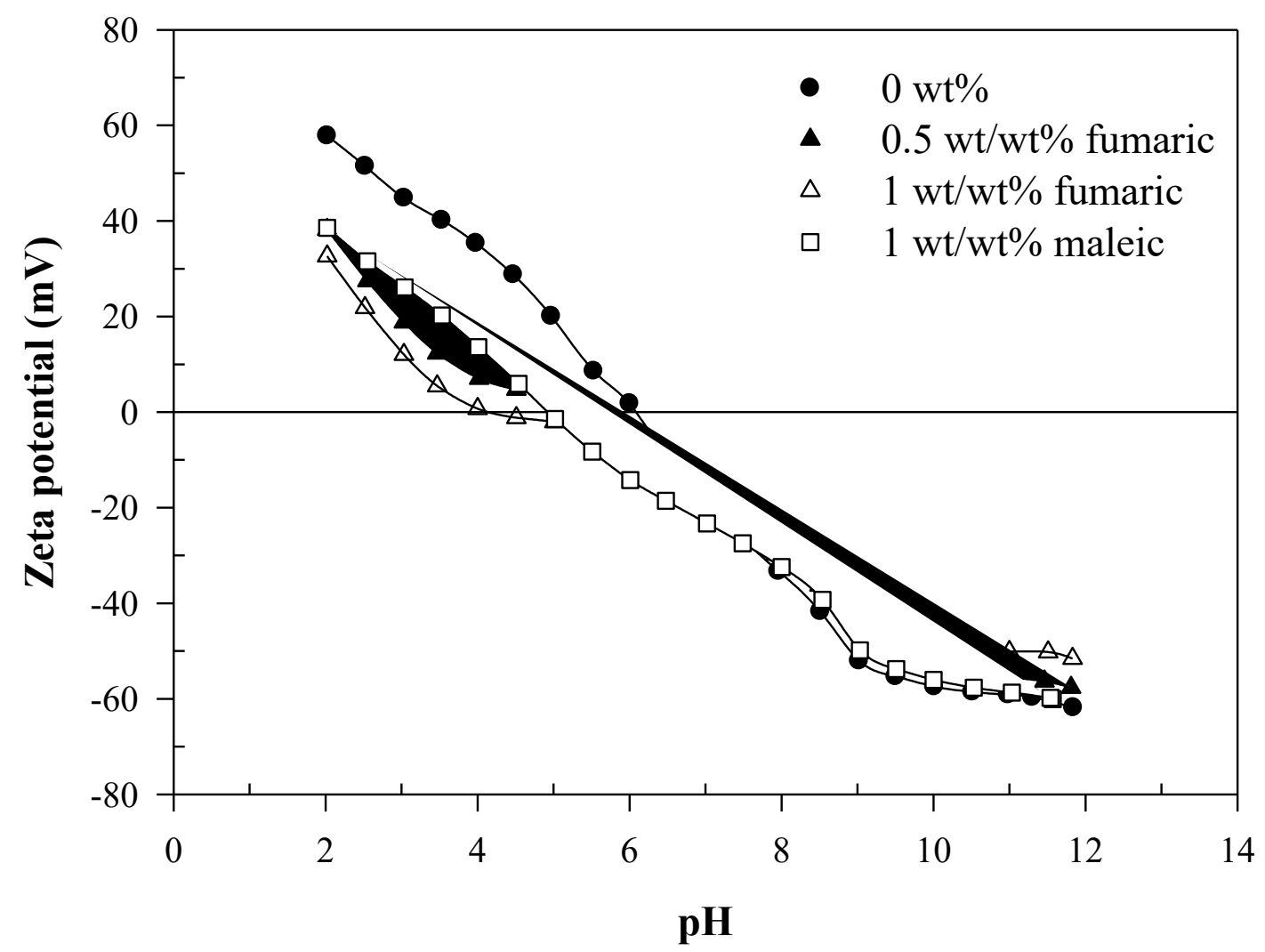

Figure 6 Zeta potential of SG TO200 titania particles as a function of $\mathrm{pH}$ in the absence and presence of fumaric and maleic acids.

\section{Direct Force Measurements between ALD Titania Surfaces}

\section{Bare ALD Titania Surfaces}

We first examined the surface forces between titania surfaces in the absence of dicarboxylic acids. The force normalised by the radius of the probe (F/R) measured on approach between ALD titania surfaces in $10^{-3} \mathrm{M} \mathrm{NaCl}$ at $\mathrm{pH} 4.5$ is presented in Figure 7. All the AFM measurements were made in the presence of $10^{-3} \mathrm{M} \mathrm{NaCl}$ to control the ionic strength. A monotonically attractive force is 
discernible from the baseline at a separation of $>30 \mathrm{~nm}$. At approximately $15 \mathrm{~nm}$, the surfaces jumped into contact due to the attraction causing an instability of the cantilever as indicated by the arrow. The attraction is attributed to attractive van der Waals (vdW) forces and is well described by a nonretarded Hamaker constant $\left(5.6 \times 10^{-20} \mathrm{~J}\right)$ calculated using the method of Walsh et al. ${ }^{14}$. This is consistent with the value previously used by Larson et al. ${ }^{28}$.

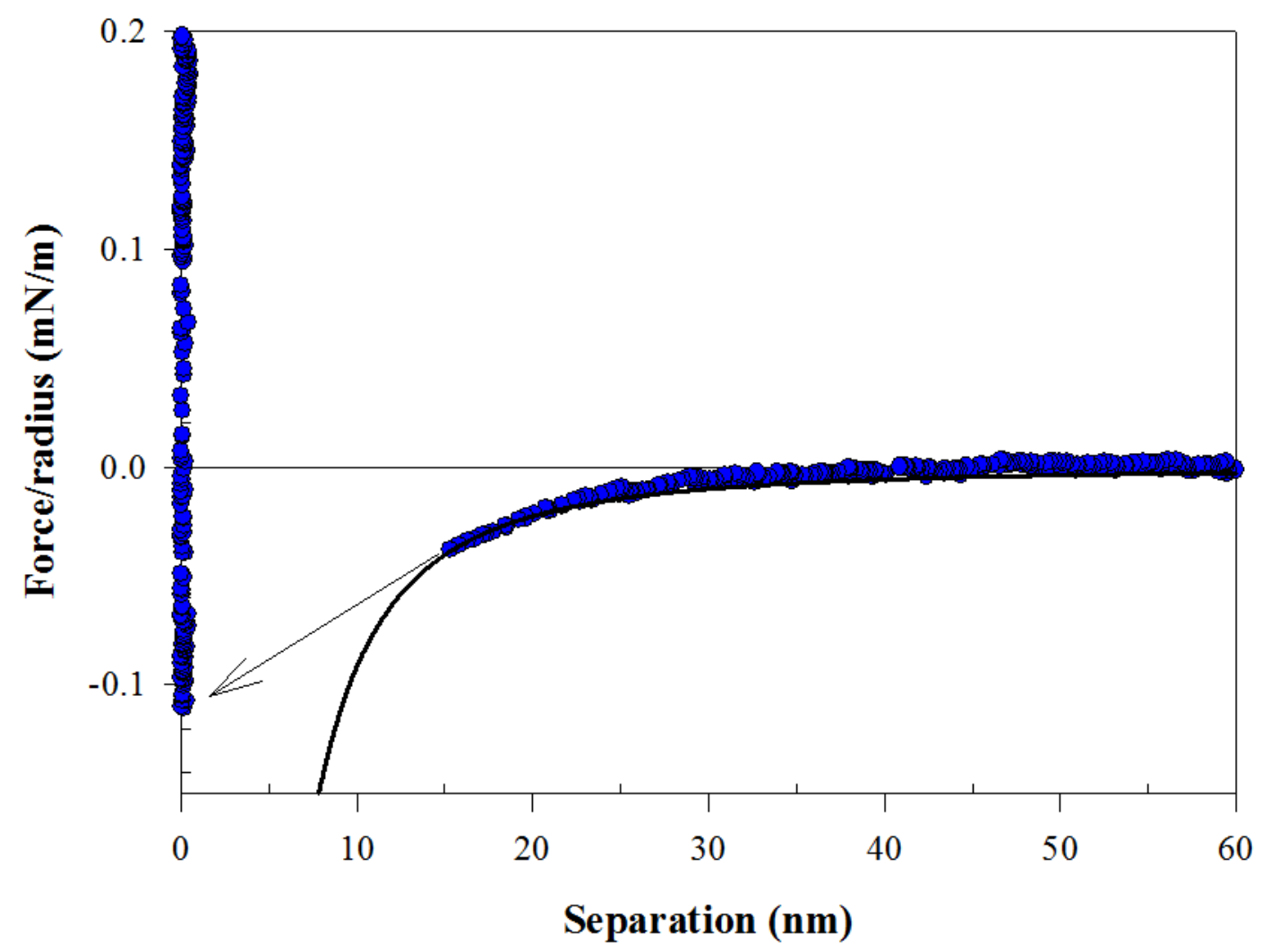

Figure 7 Measured F/R versus separation (circles) between ALD titania surfaces in $10^{-3} \mathrm{M} \mathrm{NaCl}$ at $\mathrm{pH}$ 4.5. The theoretical van der Waals interaction (unbroken line) modelled using a non-retarded Hamaker constant of $5.6 \times 20^{-10} \mathrm{~J}$ is shown for comparison.

The normalized forces measured upon approach and separation between ALD titania surfaces at pH 2.8 in $10^{-3} \mathrm{M} \mathrm{NaCl}$ are shown in Figure 8 and in the inset on a log-linear scale. At pH 2.8, a double-layer repulsion is evident. No hysteresis was observed between the approach and separation force curves, i.e., an absence of adhesion. This is consistent with earlier studies on titania surfaces ${ }^{14}$. The lack of an adhesion is attributed to a combination of a repulsive hydration force and surface roughness ${ }^{13}$. 


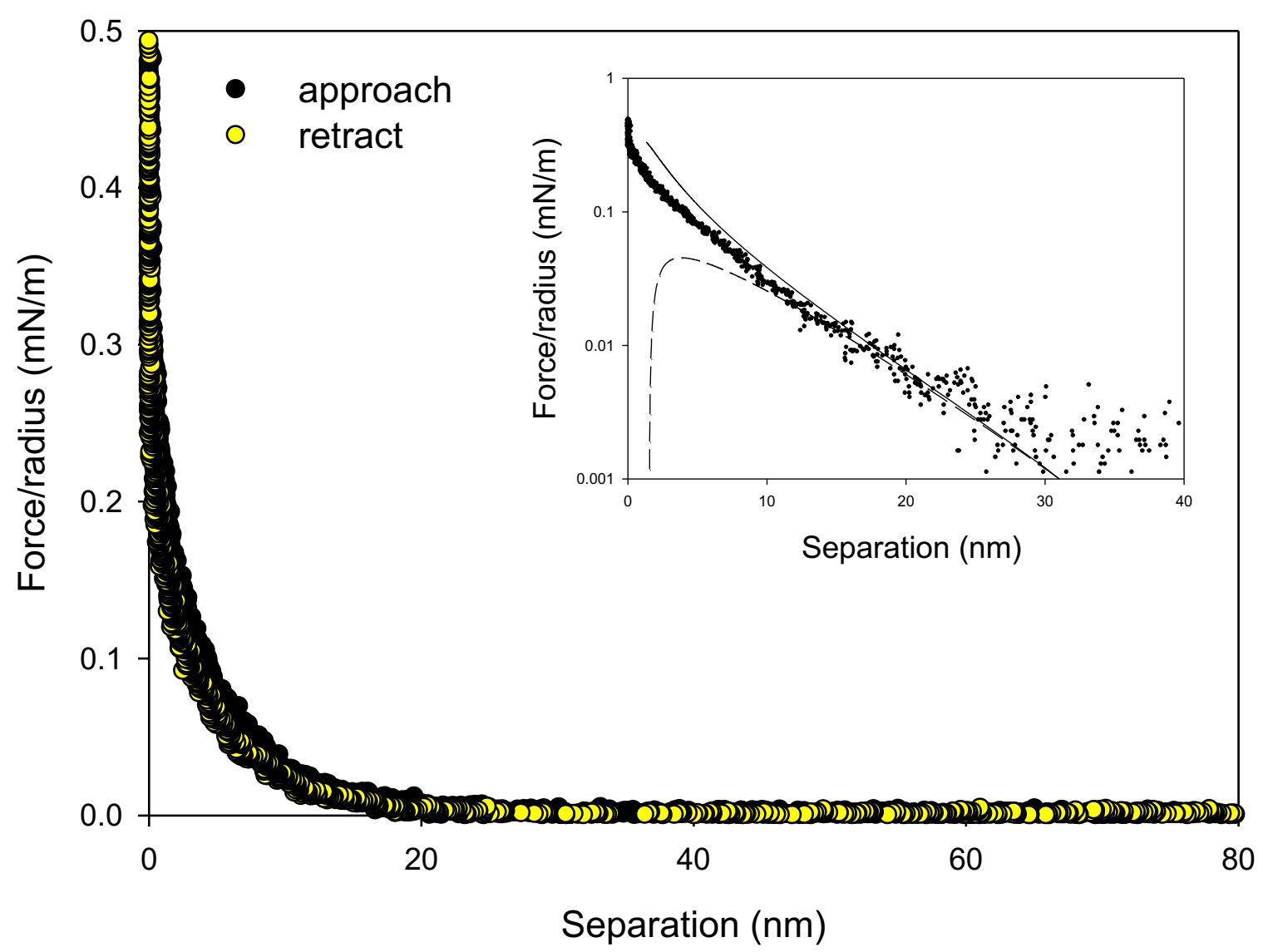

Figure 8 Measured F/R versus separation measured on approach and withdrawal between ALD titania surfaces at $\mathrm{pH} 2.8$ in $10^{-3} \mathrm{M} \mathrm{NaCl}$. Inset: The approach data plotted on a log-linear scale compared to a theoretical fit to the data using Derjaguin Landau Verwey Overbeek (DLVO) $)^{29}$ theory (line) ( $\psi_{0}=+12.4 \mathrm{mV}$, $\left.\kappa^{-1}=7.2 \mathrm{~nm}\right)$

\section{ALD Titania Surfaces in the Presence of Fumaric Acid}

We are interested in studying the surface forces in the $\mathrm{pH}$ region where the dicarboxylic acids adsorb to the surface, thus the AFM experiments were conducted at $\mathrm{pH}$ values ranging between 2 and 3 . In this study, the need for ultrasmooth surfaces becomes apparent because the dicarboxylic acids used are less than a nanometre in size. The normalized force curves measured on approach between ALD titania surfaces at various concentrations of fumaric acid between $\mathrm{pH} 2$ and 3 are illustrated on loglinear and linear-linear scales in Figures 9 and 10 respectively. At low concentrations of fumaric acid, the forces between titania surfaces were repulsive all the way into contact. By increasing the fumaric acid concentration from $10^{-4} \mathrm{M}$ to $10^{-3} \mathrm{M}$, the surface potential decreased from $+13 \mathrm{mV}$ to $+7.7 \mathrm{mV}$ and the repulsion diminished. Note that the surface potential of the ALD titania surface in the absence of these dicarboxylic acids at $\sim \mathrm{pH} 2$ is approximately $+13 \mathrm{mV}$. The Hamaker constant used to fit the experimental data in the presence of fumaric acid was $1.0 \times 10^{-21} \mathrm{~J}$ considering there was a layer of 
adsorbed fumaric acid on the titania surfaces. This reduced Hamaker constant will be used when dicarboxylic acids have adsorbed to the surface. At a fumaric acid concentration of $0.01 \mathrm{M}$, a weak repulsion was observed starting at a separation of $\sim 8 \mathrm{~nm}$ before the tip and substrate came into contact. Very similar forces were measured for a fumaric acid concentration of $0.03 \mathrm{M}$.

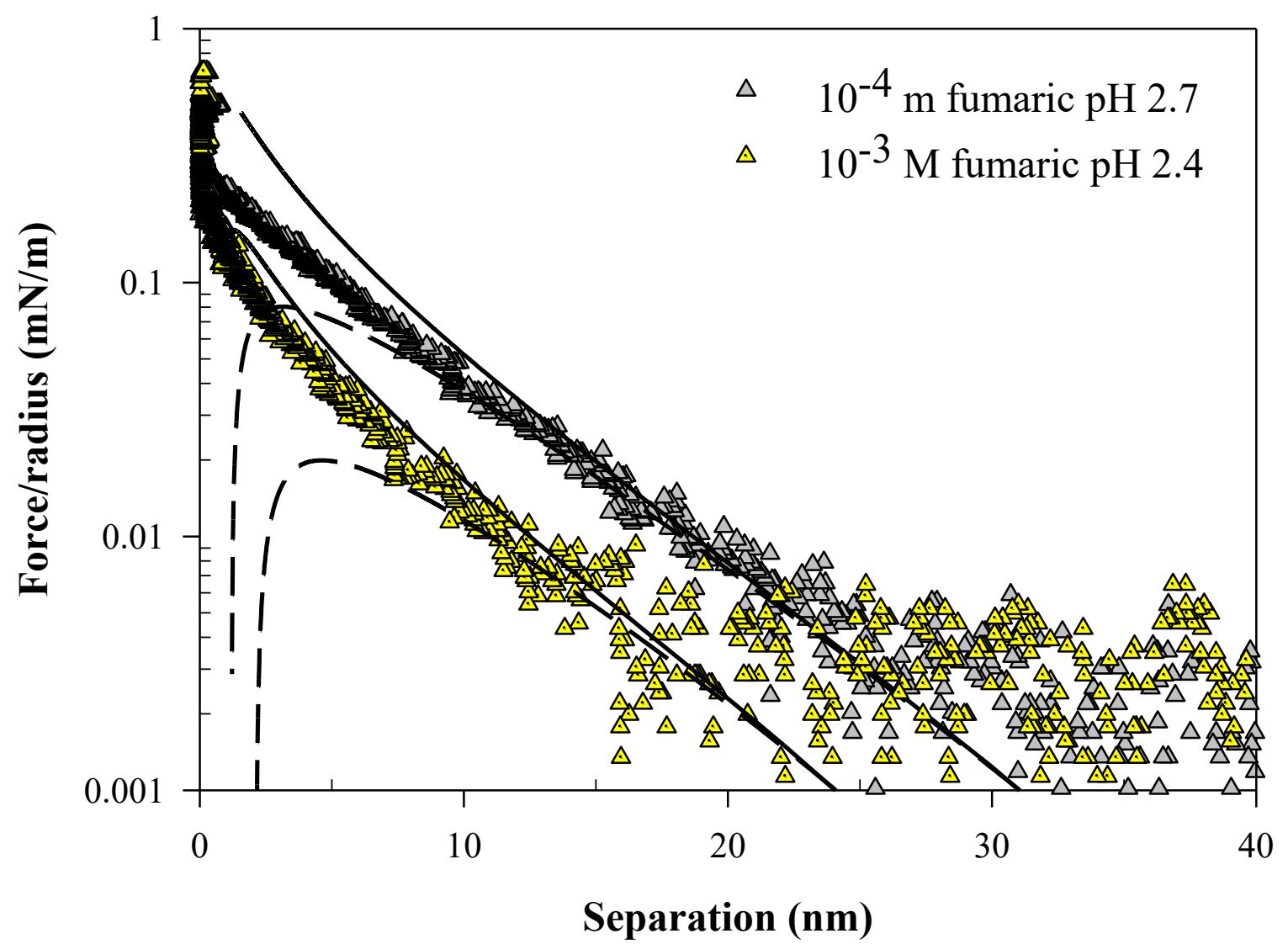

Figure 9 Normalized force versus separation measured on approach between ALD titania surfaces in $10^{-3} \mathrm{M}$ $\mathrm{NaCl}$ and $10^{-4} \mathrm{M}$ or $10^{-3} \mathrm{M}$ fumaric acid at $\sim \mathrm{pH} 2$ on a logarithmic scale compared to DLVO fits to the data using constant charge (solid line) and constant potential (dashed line) boundary conditions . Fitting parameters in $10^{-3} \mathrm{M}$ fumaric acid used: $\psi_{0}=+7.7 \mathrm{mV}, \kappa^{-1}=5.7 \mathrm{~nm}$; and $10^{-4} \mathrm{M}$ fumaric acid: $\psi_{0}=+13 \mathrm{mV}, \kappa^{-1}=5.8$ $\mathrm{nm}$. 


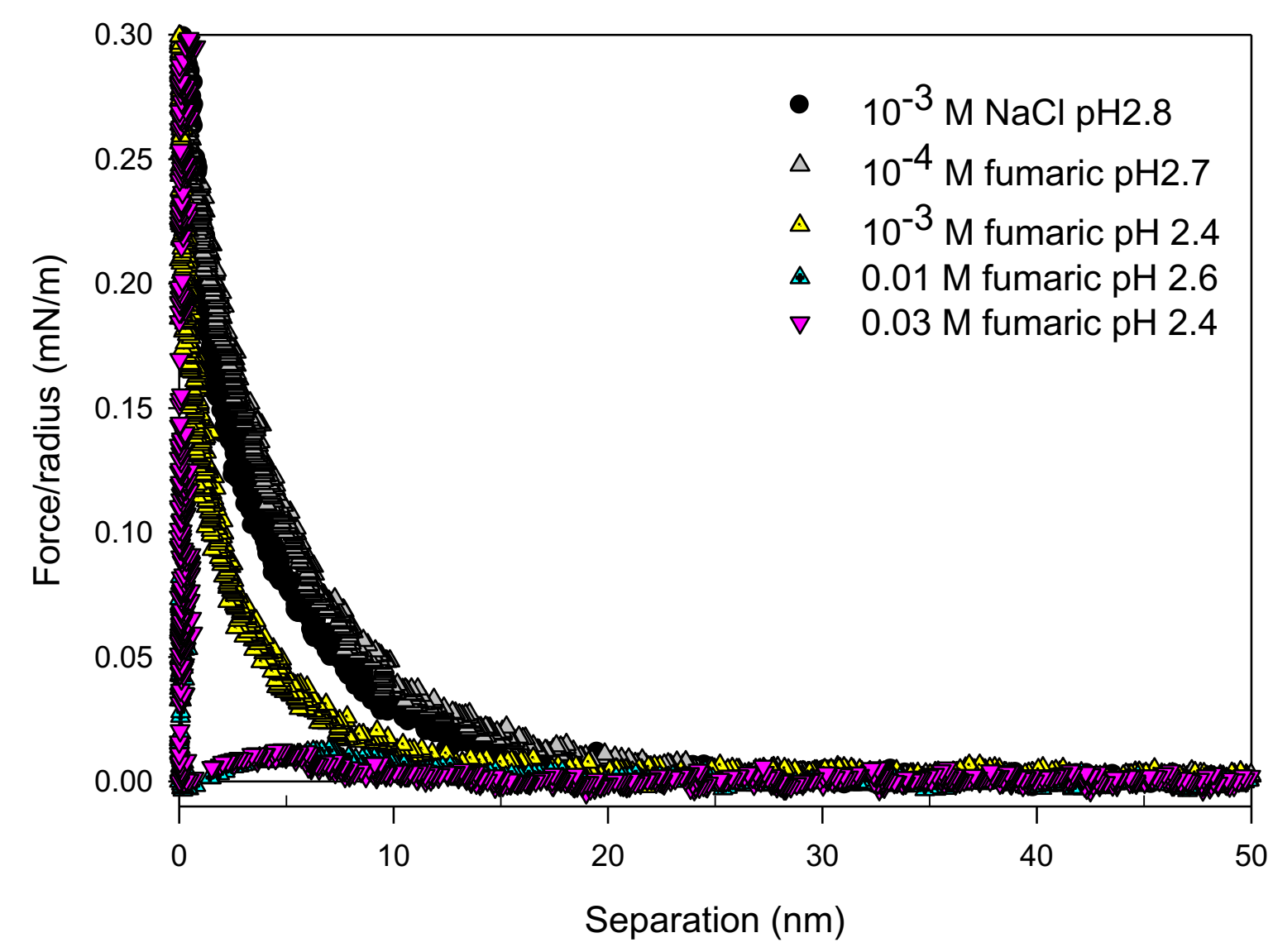

Figure 10 Normalized force versus separation measured on approach between ALD titania surfaces at various concentrations of fumaric acid at $\mathrm{pH} 2-3$. At low concentrations of fumaric acid, the titania surfaces were strongly repulsive upon approach. The repulsion diminished with increasing acid concentration.

The normalized force curves measured upon separation as a function of fumaric acid concentration over the $\mathrm{pH}$ range of 2-3 are presented in Figure 11. At low concentrations $(<0.01 \mathrm{M})$, there was no adhesion when the surfaces were separated. Only upon increasing the concentration (>0.01 M) was adhesion observed. When the adhesion was overcome the surfaces jumped apart to a non-deflected position as indicated by the arrows in Figure 11. 


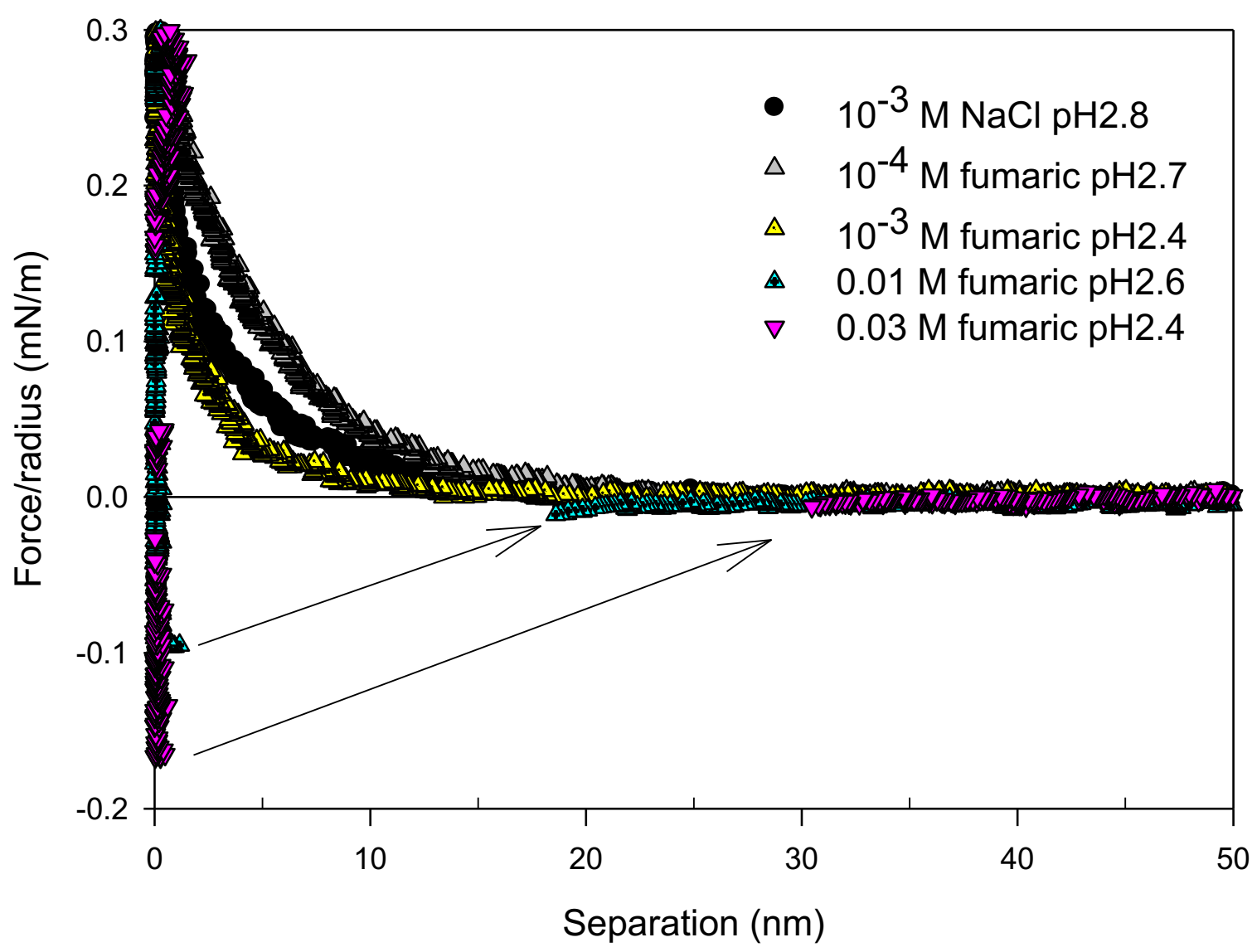

Figure 11 Normalized force versus separation measured upon separation between ALD titania surfaces at various concentrations of fumaric acid at $\mathrm{pH} 2-3$. No adhesion was seen at concentrations of $10^{-4} \mathrm{M}$ or $10^{-3}$ $\mathrm{M}$ The adhesion (as characterized by the minimum in the force) was greater at a fumaric acid concentration of $0.03 \mathrm{M}$ compared to $0.01 \mathrm{M}$.

\section{ALD Titania Surfaces in the Presence of Maleic Acid}

The normalized forces measured on approach between titania surfaces in the presence of maleic acid at $\mathrm{pH}$ in the range of $2-3$ are shown in Figure 12 . At $10^{-3} \mathrm{M}$ maleic acid, the surface potential was +14.6 $\mathrm{mV}$ compared to $+7.7 \mathrm{mV}$ at the same concentration of fumaric acid. The interaction upon approach is somewhat similar to that of fumaric acid whereby at low concentrations, the repulsive force was progressively reduced. Yet, they differ in that at $0.01 \mathrm{M}$, the forces were still repulsive for maleic acid. At high concentration, there was no strong attraction, even in the last few nanometers. It is worthwhile to note that in spite of the difference in molecular architecture, the approach forces in the maleic acid system did not differ from that of fumaric acid at $\sim \mathrm{pH} 2$. On the contrary, the adhesion forces were markedly different in that there was no significant adhesion in the titania-maleic acid system (compare the inset of Figure 12 with Figure 11). 


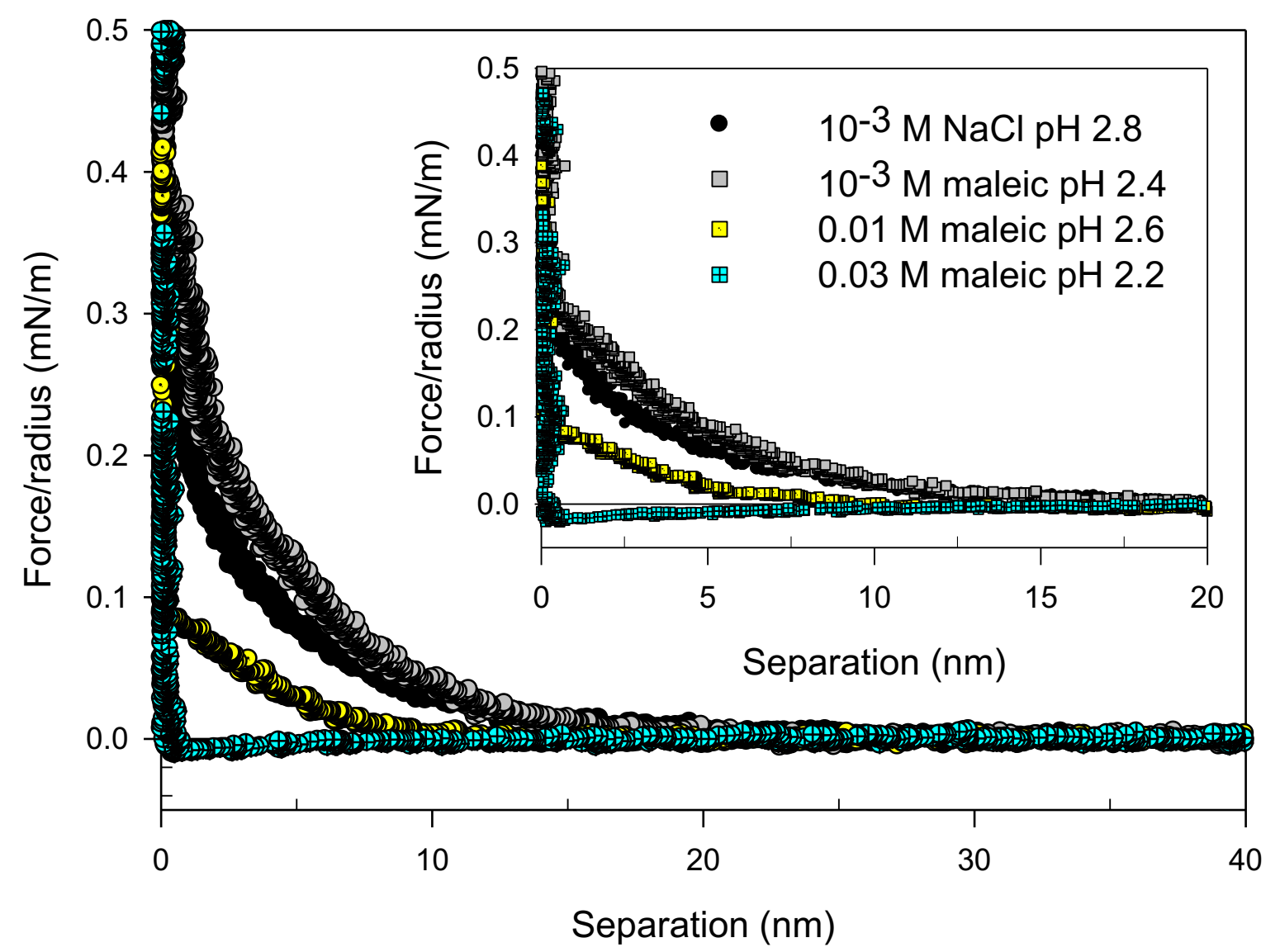

Figure 12 Normalized forces versus separation measured on approach between ALD titania surfaces at various concentrations of maleic acid at $\mathrm{pH}$ 2-3. Inset shows the forces upon separation. No significant adhesion was observed in the titania-maleic acid system.

\section{Rheology of Titania Particles in the Presence of Fumaric and Maleic Acids}

\section{Yield Stress}

The yield stress as a function of $\mathrm{pH}$ for the pure SG TO200 titania system was somewhat parabolic in shape as shown in Figure 13 with the maximum yield stress, $\tau_{\max }$ of $500 \mathrm{~Pa}$ at $\sim \mathrm{pH}$ 6.3. This $\mathrm{pH}$

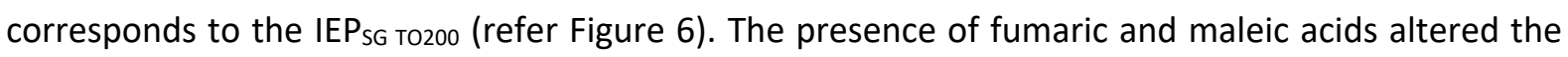
yield stress of the SG TO200 titania dispersions significantly as illustrated in Figure 13. In the presence of maleic acid the yield stress generally decreased with decreasing $\mathrm{pH}$ and was notably different for $0.5 \mathrm{wt} \%$ and $0.8 \mathrm{wt} \%$ fumaric acid. At $0.5 \mathrm{wt} \%$ fumaric acid, the yield stress was almost constant from $\mathrm{pH} 4$ to $\mathrm{pH} 10$ and at $0.8 \mathrm{wt} \%$ fumaric acid the yield stress was almost constant from $\mathrm{pH} 3$ to $\mathrm{pH} 11$. 


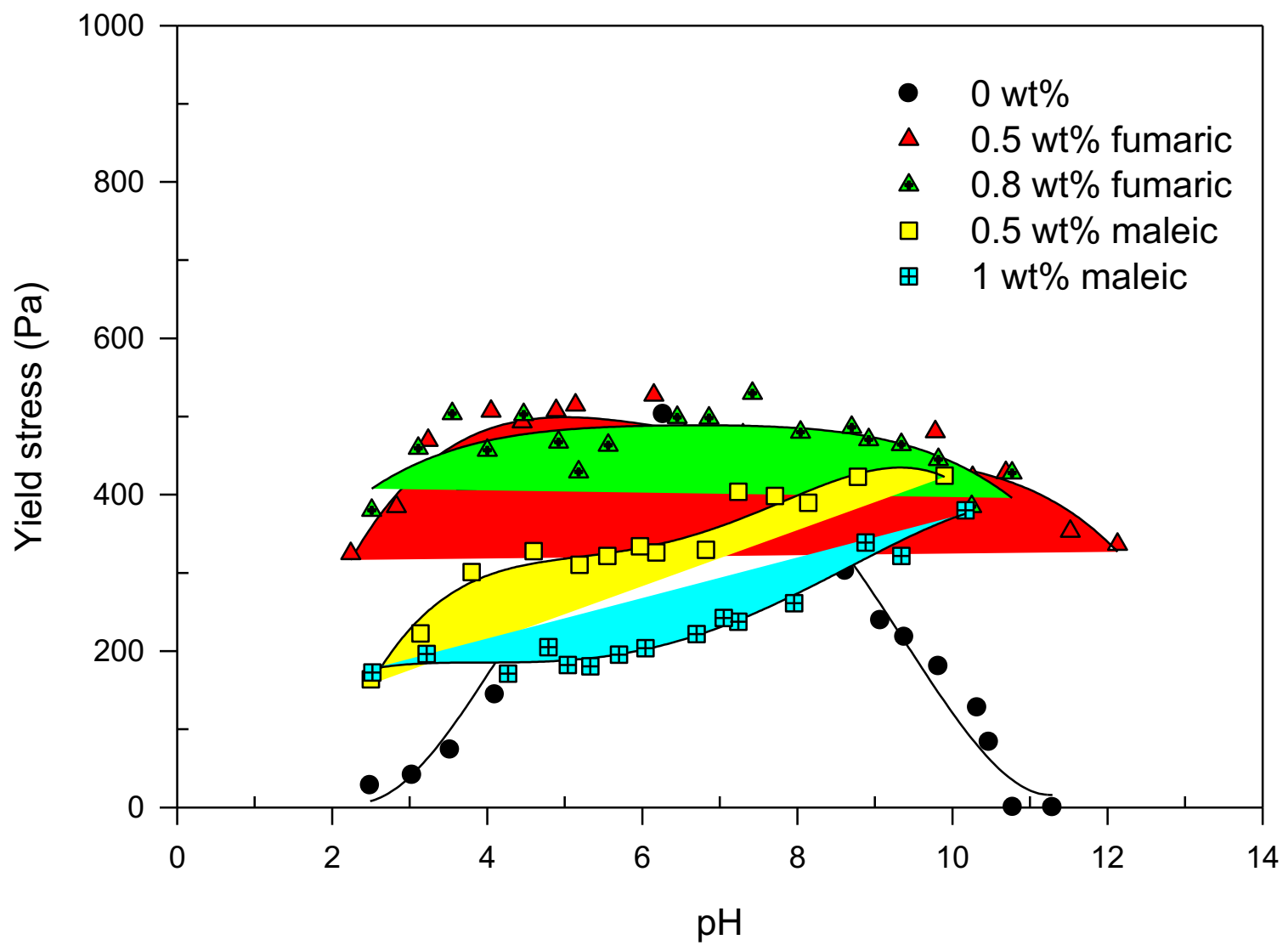

Figure 13 Yield stress versus $\mathrm{pH}$ for SG TO200 titania suspensions at various concentrations of fumaric and maleic acids. The ionic strength of the pure titania dispersion and that in the presence of fumaric and maleic acids was on the order of $0.04 \mathrm{M}$ and $0.12 \mathrm{M}$ respectively. The lines are to guide the eye. The yield stress curves are notably different in the presence of fumaric and maleic acids. 


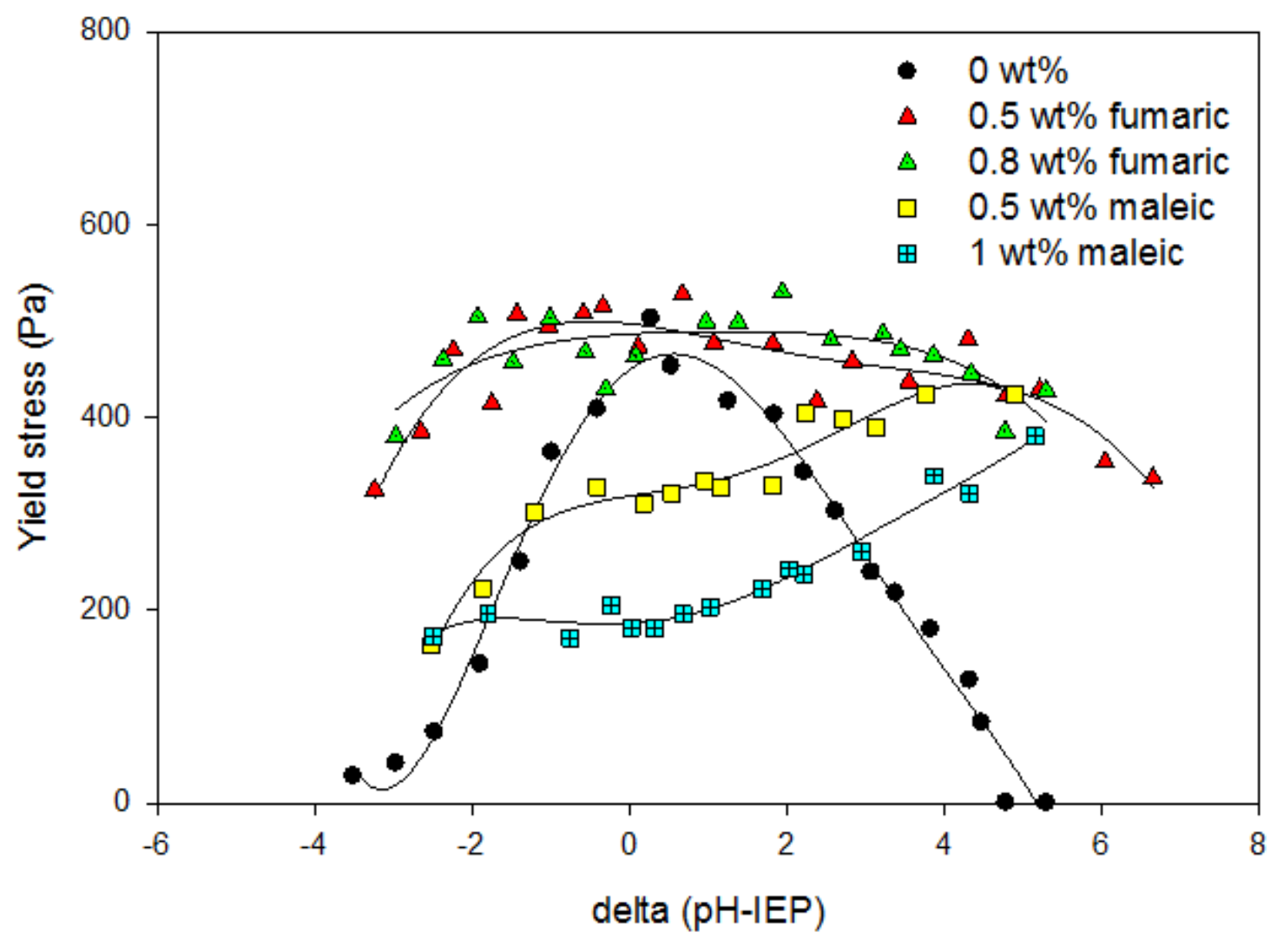




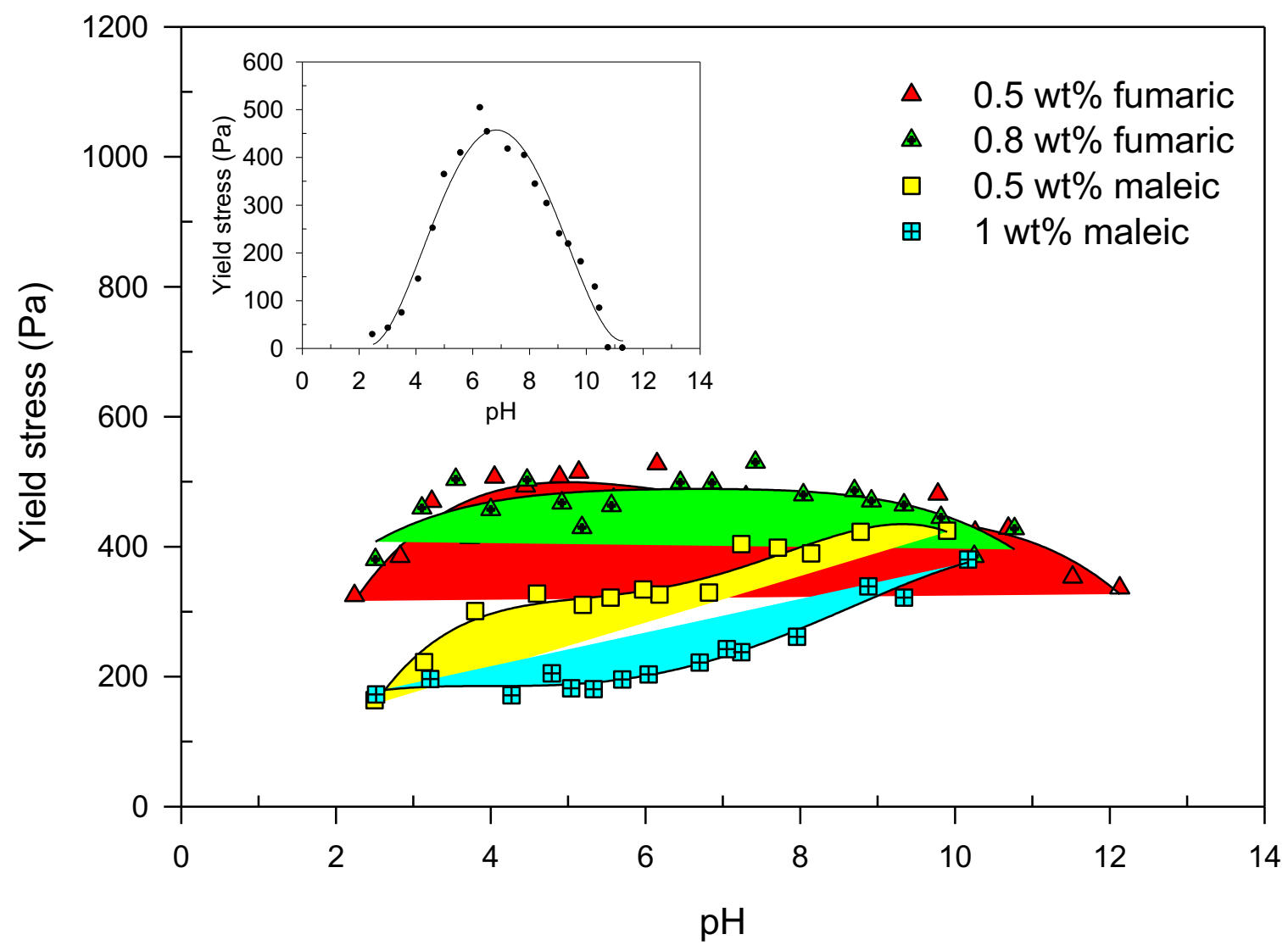

\section{Discussion}

\section{Surface Chemistry of Titania}

The charging behaviour of the titania surface in water is determined by amphoteric hydroxyl groups at the surface. These groups may be tethered to the titanium atom or to an oxygen atom. The acidity of these groups will be dependent on the tethering, the local crystal structure and the proximity of neighbouring hydroxyl groups. Neighbouring hydroxyl groups can interact both by hydrogen bonding and by charge interactions. Further, whilst all sites can participate in physical adsorption only some sites will be suitable for chemisorption (surface complexation). Thus the hydroxyl groups at the titania surface present a multitude of sites for adsorption and these will vary along with the IEP for titania 
surfaces of different roughness and crystal structure. ${ }^{30}$ This highlights that the IEP is the $\mathrm{pH}$ at which the negatively charged sites balance the positively charged sites and that $\mathrm{pH}$ values above but still in the vicinity of the IEP there will still be positively charged sites for adsorbing anions and the converse will be true below the IEP.

\section{Adsorption of fumaric and maleic acids to titania}

Fumaric and maleic acids can exist in three different protonation states depending on the $\mathrm{pH}$ of the solution: neutral $\left(\mathrm{H}_{2} \mathrm{X}\right)$, monoanion $\left(\mathrm{HX}^{-}\right)$and deprotonated, dianion $\left(\mathrm{X}^{2-}\right)$. Fumaric acid has a $\mathrm{p} K \mathrm{a}_{1}$ of 3.03 and a $\mathrm{pKa}_{2}$ of 4.54 while maleic acid has a $\mathrm{pKa}_{1}$ of 1.83 and a $\mathrm{pKa}_{2}$ of $6.07^{6}$, though the $\mathrm{pH}$ dependent ionisation of a molecule adsorbed to a surface will be substantially different (as discussed below). At the $\mathrm{pH}$ range employed in this work $(\mathrm{pH} 2-3)$, which is lower than the $\mathrm{pKa}_{1}$ of the acid, the majority of the fumaric acid molecules in solution will be in the neutral state whereas the majority of the maleic acid molecules will be present in solution as the monoanion.

Various workers have studied how LMW carboxylic acids adsorb to mineral surfaces. Hwang and Lenhart ${ }^{31}$ used attenuated total reflectance Fourier-transform infrared (ATR-FTIR) to show that fumaric acid adsorbed in its fully deprotonated form even at low $\mathrm{pH}(\mathrm{pH} 3.5)$. This is also observed in the work of Dobson and Mcquillan ${ }^{32}$. Hwang and Lenhart ${ }^{31}$ noted that the IR spectra of adsorbed fumaric acid only pointed to an outer-sphere mode of complexation. Outer-sphere complexation is where the interactions between the adsorbing organic acid and surface are mediated by the presence of surface bound water or hydroxyl groups via hydrogen bonding and/or simple electrostatic interactions without any direct bonding with the surface metal ion ${ }^{33}$. Yao and Yeh ${ }^{34}$ reported at similar initial concentrations, fumaric acid displayed a stronger ionic-strength dependency compared to its cis counterpart which is in line with the outer-sphere adsorption mode.

Maleic acid showed similar behaviour to its trans counterpart, also adsorbing in its fully deprotonated

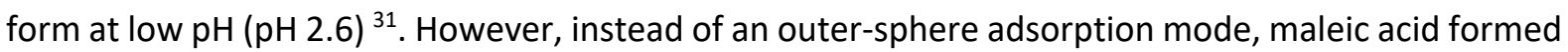
an inner-sphere complex with the metal oxide surface at low $\mathrm{pH}^{31}$. Inner-sphere complexation occurs when the adsorbing organic acid directly bonds with a surface metal ion ${ }^{33}$. With increasing $\mathrm{pH}$, the adsorption of maleic acid transitioned to an outer-sphere complex.

Dissociative adsorption is an important consideration here as the second deprotonated carboxylic group of the adsorbed fumaric acid will extend into the bulk solution due to the trans configuration 
and the double carbon bond that restricts the molecular flexibility. Therefore, it is impossible for both of the carboxylate groups of fumaric acid to adsorb onto the same surface. As fumaric acid adsorbs via one carboxylate group, the second free carboxylate group has the potential to adsorb to a second particle provided it is close enough. Therefore it has been proposed that the adsorption of fumaric acid aggregates hematite particles by "bridging" neighbouring particles ${ }^{31}$ which is in agreement with the highly directed bridging hypothesis of Leong ${ }^{6}$. Conversely, both carboxylate groups in maleic acid upon adsorption will face the same particle as the two functional groups are on the same side of the planar ethylene unit. This adsorption configuration has led to various studies ${ }^{6,35}$ reporting maleic acid as a steric agent (providing a short range structural repulsion). Figure 13 shows a schematic representation of the expected adsorption configuration of fumaric and maleic acids.

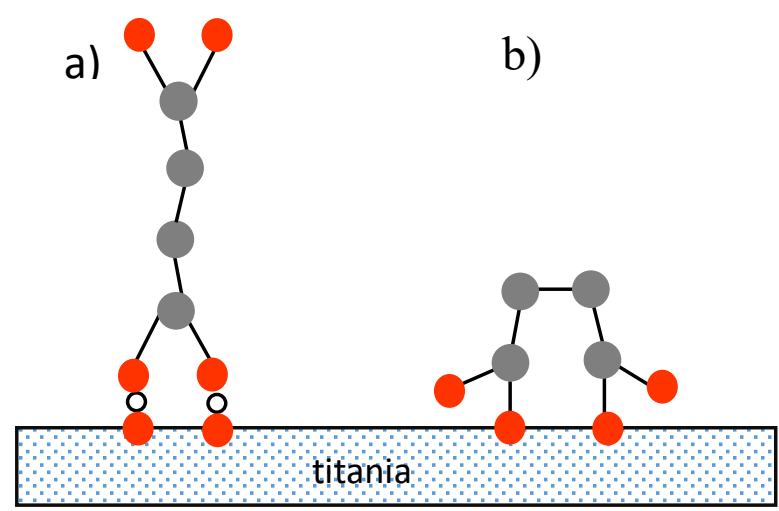

Figure 14 Schematic representation of the adsorption configuration of (a) fumaric and (b) maleic acids at the titania/water interface. Note that the red, grey and white balls represent the oxygen, carbon and hydrogen atoms respectively. Fumaric acid has a trans configuration and therefore, adsorbtion by one acid group results in the other group facing away from the surface. On the other hand, both the carboxylate groups in the maleic acid molecule are able to interact with the same surface as the two functional groups are on the same side of the planar ethylene unit.

\section{Direct Force Measurements between ALD Titania Surfaces}

\section{Bare ALD Titania Surface}

Larson et al. ${ }^{28}$ measured the interaction between a rutile colloid probe and substrate in an aqueous solution of $10^{-4} \mathrm{M} \mathrm{KNO}_{3}$. They used an experimental Hamaker constant of $6 \pm 2 \times 10^{-20} \mathrm{~J}$ to fit the force curves at the isoelectric point (IEP) of their titania surface. This was found to be comparable to the value of $7 \pm 1 \times 10^{-20} \mathrm{~J}$ calculated from spectroscopic data on rutile titania using Lifshitz theory. However, one major shortcoming of this study ${ }^{28}$ is that the aspherical colloid probe used was rough 
and had deep crevices on the surface which severely affected the determination of the effective area of the colloid probe interacting with the substrate and the true surface separation and thus complicates interpretation of the data. In our study, we have overcome this surface roughness limitation by preparing titania surfaces using the ALD process. The fact that a non-retarded Hamaker constant of $5.6 \times 10^{-20} \mathrm{~J}$ was found to fit the experimental data at $\mathrm{pH} 4.5$ indicates that the surface must be near neutral, i.e., close to the isoelectric point. At the IEP, there is no electrostatic repulsion and thus the van der Waals ( $\mathrm{vdW}$ ) attractive force is evident. The measured IEP $\mathrm{ALD}_{\text {of }} \sim \mathrm{pH} 4.5$ indicates that the ALD process has produced a titania film which has an IEP substantially different to the silica substrate which is $\sim \mathrm{pH} 2^{24,26,36,37,38}$. The IEP of the ALD titania falls within the range of values reported in the literature for uncontaminated titania surfaces $26,27,38$. In other studies using similar ALD titania surfaces, Walsh found that the IEP ALD obtained via zeta potential measurements was $\sim \mathrm{pH} 5{ }^{39}$ which is very close to the IEPALD measured in this study ( $\mathrm{pH} 4.5)$. X-ray diffraction (XRD) measurements on the ALD substrate indicate that the ALD titania surfaces were amorphous in nature ${ }^{39}$. The crystal structure is known to influence the IEP of titania surfaces so we might expect a difference between the amorphous surface and rutile or anatase surfaces.

The surface forces between titania surfaces in $10^{-3} \mathrm{M} \mathrm{NaCl}$ at $\mathrm{pH} 2.8$ are identical on approach and upon separation and the expected dispersion forces are not evident. This has been attributed to the presence of short-range repulsive forces such as hydration forces normally reported in the case of silica surfaces ${ }^{15}$. However, as hydration forces are of very short range, an attraction should still be evident in the titania system as the dispersion forces are much stronger and therefore extend further from the titania surfaces than would be expected from silica surfaces. The presence of a strong van der Waals attraction in the absence of surface charge that is no longer apparent when the surfaces become charged has been attributed to surface roughness ${ }^{13}$.

\section{ALD Titania Surfaces in the Presence of Fumaric and Maleic Acids}

In this study, a repulsion was measured between the approaching titania surfaces in the presence of fumaric acid at $\mathrm{pH} 2-3$ (see Figure 10$)$. At low surface coverage $\left(10^{-4} \mathrm{M}\right.$ and $10^{-3} \mathrm{M}$ fumaric acid), the interaction was systematically reduced with increasing surface coverage. Upon increasing the concentration to $0.01 \mathrm{M}$, the repulsion was almost insignificant. Both the surface forces and the zeta potential show that the adsorption of fumaric acid reduced the surface charge of the titania surfaces. That is, as the amount of adsorbed fumaric acid increased, the surface charge decreased and the repulsive force present when the two surfaces approached was reduced. The vdW interaction is 
largely unaffected by experimental conditions such as ionic strength and $\mathrm{pH}$ but adsorption of molecules to the surface will reduce the magnitude of the attraction both by screening the dispersion forces and through other effects such as steric repulsion.

The forces measured when the surfaces are separated can be employed to probe the nature of the adsorbed layer. In the case of adsorbed polymer, the adhesion forces are often associated with a series of steps or secondary adhesions which are a signature for the "pulling" of polymer chains that are bridging between the surfaces ${ }^{10,12}$. These polymer extension forces can extend to thousands of nanometers depending on the molecular weight of the adsorbed polymer ${ }^{10}$. In this study, we did not find such pull-off forces which rules out polymer contamination as the cause of the behaviour observed in this study (noting that any bridging induced by the dicarboxylic acids will be too shortranged to be directly detected).

The cis and trans isomers have different ionisation properties in solution but the evidence so far points to the full ionization of both acids upon adsorption, as discussed above. Also, we have seen that the surface excess is similar (see Table 1). The significant difference in the adhesion measured between maleic and fumaric acids must therefore have an explanation in the physical architecture of the molecules. Maleic acid, being in a cis configuration upon adsorption orients such that both carboxylic groups face the same surface whereas fumaric acid adsorbs via a single carboxylate group with the other carboxylate group oriented away from the surface into solution (see Figure 13). The difference in the measured adhesion is consistent with the view that maleic acid provides a steric repulsion whereas fumaric acid promotes adhesion through molecular bridging whereby the carboxylate acid group that orients into solution bridges a second titania surface.

\section{Rheology of Titania Particles in the Presence of Fumaric and Maleic Acids}

\section{Zeta Potential}

Dicarboxylic acids are known to adsorb to colloidal particles 9, 40,41, 42 altering the zeta potential and shifting the IEP $26,27,38$. Titania particles have been reported to display a range of IEPs, depending on the crystal structure and surface impurities from the preparation process ${ }^{20}$. Washing to remove impurities can result in significant shifts in the IEP. Kosmulski and Rosenholm ${ }^{43}$ reported anatase particles with an IEP at $\mathrm{pH}<3.4$ prior to washing had an IEP at $\mathrm{pH} 5.9$ after washing. The increase in IEP post-washing was attributed to the removal of a substantial amount of multivalent ions from the 
titania surfaces. In another study ${ }^{20}$, an unwashed batch of anatase titania particles displayed an IEP of $\sim \mathrm{pH} 5$, this increased to $\sim \mathrm{pH} 6.8$ following washing. These studies show that the IEP obtained via zeta potential measurement is a good measure of the surface cleanliness of the colloid particles. Negligible changes to the IEP before and after washing confirms the cleanliness of our SGT O200 particles.

As the dicarboxylic acids are charged over a wide range of $\mathrm{pH}$, the zeta potential-pH behaviour serves as a good indication of the adsorption behaviour of the acids. Above the IEP $\mathrm{SG}_{\mathrm{SO}}$ To0 little adsorption occurred as both the titania surfaces and the acids were negatively charged. Below the IEPSG TO200 the adsorption of fumaric and maleic acids shifted the IEP to a lower $\mathrm{pH}$ due to anion adsorption ${ }^{44,45}$. The shift of the IEP was as much as $0.5 \mathrm{pH}$ units in $0.5 \mathrm{wt} \%$ fumaric acid and $1 \mathrm{pH}$ unit at the same concentration of maleic acid. At pH 2, the zeta potential in the presence of fumaric and maleic acids did not converge to that of the pure titania system. This shows that at low $\mathrm{pH}$, acids remain adsorbed to the titania/water interface and that they carry a negative charge despite the species in solution becoming protonated.

\section{Yield Stress}

The maximum yield stress, $\tau_{\max }$ for the pure SGT O200 titania suspensions occurred at the IEP. At the IEP, the electrostatic repulsion is absent and the van der waals attraction is expected to dominate. We therefore attribute the $\tau_{\max }$ to van der Waals forces. Away from the IEP, the surfaces acquire a charge and the electrostatic repulsion becomes significant leading the the yield stress to diminish and eventually disappear.

The rheology of the titania dispersions underwent a striking change upon the introduction of the carboxylic acids. At high $\mathrm{pH}$ the yield stress for both maleic and fumaric acids was significantly higher than that for bare titania. At this $\mathrm{pH}$ the acids do not adsorb to the surface. The increase in yield stress above the IEP is ascribed to the increase in ionic strength, which screens the electrostatic repulsion between the surfaces. With the electrostatic repulsion highly screened the magnitude of the yield stress is similar to that of the pure titania system at the IEP, showing that the influence of the electrostatic repulsion on the yield stress has been completely removed.

For maleic acid as the $\mathrm{pH}$ is decreased the yield stress steadily decreases with the decrease being steeper at higher concentration. This is accompanied by an increase in adsorption of maleic acid at 
low pH (see Figure 5). Adsorption decreases the surface charge and consequently the electrostatic repulsion and this can lead to an increase in yield stress. However, in this case, the influence of the electrostatic repulsion has already been nullified by the ionic strength so the reduction in charge could not further increase the yield stress. Yet, a reduction in yield stress is seen upon the adsorption of maleic acid. This can be attributed to two effects. Firstly, the adsorption of maleic acid decreases the Hamaker constant of the system which reduces the attraction between the surfaces. Secondly, maleic acid is thought to sterically hinder the interaction between titania particles in contact. The net effect is a reduction in adhesion between titania surfaces and correspondingly a decrease the yield stress. This is borne out in the surface forces where in the titania-maleic acid system, the adhesion is minimal.

The yield stress as a function of $\mathrm{pH}$ in the presence of fumaric acid is substantially different to maleic acid. The titania dispersions remained flocculated at the entire $\mathrm{pH}$ range investigated $(\mathrm{pH} 2-12)$. At very high $\mathrm{pH}$ the yield stress is similar to that of maleic acid. This is attributed to the increase in ionic strength shielding the electrostatic repulsion which leads to adhesion and a yield stress similar to that of the bare titania at the IEP. The difference is that as the $\mathrm{pH}$ is reduced the yield stress for fumaric acid is rather constant, whereas maleic acid showed a decreasing yield stress. In both cases the drop in $\mathrm{pH}$ is accompanied by an increase in adsorption. Correspondingly, the adsorbed species are charged and will therefore reduce the surface potential. However the influence of the electrostatic forces has already been nullified by the ionic strength. As such, adsorption of fumaric acid will reduce the Hamaker constant of the system and has the potential to give rise to a steric repulsion similarly to that observed in the maleic acid system. Why then does the yield stress and the surface forces show a substantially higher adhesion in fumaric acid compared to maleic acid? The difference is consistent with previous studies that argue that the molecular architecture of fumaric acid enables highly directed bridging to take place between surfaces in contact ${ }^{6,7,8,9,35,46}$. This is not seen for maleic acid due to the molecular architecture of the cis isomer. Thus, our results support the hypothesis that fumaric acid increases the yield stress by forming molecular bridges between particles.

\section{Differences between yield stress and surface forces}

The yield stress of a suspension is known to be strongly dependent on particle size, shape, solid concentration ${ }^{47}$ and the nature of adsorbing species. Differences, however minor, in the morphology of the colloid particles in addition to environmental conditions can have a huge influence on particleparticle interactions. Although the AFM pull-off forces predict an increase in attraction with increasing adsorption of fumaric acid, i.e., from $0.01 \mathrm{M}$ to $0.03 \mathrm{M}$, it is clear from Figure 13 that the $\tau_{\max }$ of the 
titania dispersions did not vary with increasing fumaric acid concentration. This discrepancy can arise due to a few reasons. Firstly, the ALD titania surfaces used in the AFM force measurements are much smoother. Secondly the force measurements cover a wider change in the surface excess of fumaric acid.

Previously fumaric acid has been shown to cause a dramatic increase in the yield stress between alumina particles ${ }^{6}$ that is far greater than the effect observed here between titania surfaces. We attribute the difference to two effects. Firstly, it has been shown that for very rough titania surfaces fumaric acid reduces the yield stress rather than increasing $i t^{48}$. Clearly a high level of surface roughness can modify or even eliminate the adhesion associated with highly directed bridging. Secondly, alumina is well-known to dissolve forming various hydrolysis products at high or low $\mathrm{pH}^{36}$. At low $\mathrm{pH}$, the presence of these positively charged hydrolysis products can lead to the formation of organic complexes with the negatively charged carboxylic acids. Johnson et al. ${ }^{35}$ attributed the yield stress increase of AKP 30 alumina suspensions at high concentration of maleate, oxalate and citrate at low $\mathrm{pH}$ to the formation of $\mathrm{Al}(\mathrm{III})$-organic gel-like precipitates. On the contrary, titania does not undergo such drastic dissolution under aqueous conditions ${ }^{21}$.

\section{Conclusion}

The presence of fumaric and maleic acids altered the rheology and surface forces between titania dramatically. Firstly, fumaric acid did not increase or decrease the yield stress of titania dispersion; instead it flattened out the yield stress curve. In contrast, maleic acid reduced the yield stress considerably. AFM measurements show an adhesion that increased with concentration in the ALD titania-fumaric acid system. This nanoscopic adhesion appears to be consistent with the previously proposed highly directed bridging mechanism which consequently gave rise to the yield stress behaviour of titania-fumaric acid dispersions. On the other hand, the cis adsorption configuration of maleic acid caused both carboxylate groups to face the same surface, thereby resulting in the absence of any adhesion. It is apparent that the differences in the rheological response of titania dispersions as a function of $\mathrm{pH}$ in the presence of fumaric and maleic acids can be linked to the measured nanoscopic adhesions and the molecular architecture of the acids. 


\section{Acknowledgement}

EJT acknowledges the use of the ALD surfaces prepared by Dr Rick Walsh, Department of Applied Mathematics, ANU. EJT acknowledges the travel funds granted by AFUW and ARNAM which made this work possible.

\section{References}

1. Israelachvili, J. N. Intermolecular and Surface Forces; 2nd ed.; Academic Press: London, 1992.

2. Israelachvili, J.; Pashley, R. The Hydrophobic Interaction is Long-range, Decaying Exponentially with Distance. Nature 1982, 300 (5890), 341-342.

3. Binnig, G.; Quate, C. F.; Gerber, C. Atomic Force Microscope. Phys. Rev. Lett. 1986, 56 (9), $930-$ 933.

4. Ralston, J.; Larson, I.; Rutland, M. W.; Feiler, A. A.; Kleijn, M. Atomic Force Microscopy and Direct Surface Force Measurements - (IUPAC technical report). Pure Appl. Chem. 2005, 77 (12), 21492170.

5. Chandramalar, A. V. M.; Lim, Y. Y.; Leong, Y. K. The Effects of cis-trans Configuration of Cyclohexane Multi-Carboxylic Acids on Colloidal Forces in Dispersions: Steric, Hydrophobic and Bridging. Colloids Surf., A 1999, 160 (3), 199-205.

6. Leong, Y. K. Molecular Configuration of Adsorbed cis- and trans-1,2-ethylene Dicarboxylic Acids and Interparticle Forces in Colloidal Dispersions. Langmuir 2002, 18 (6), 2448-2449.

7. Leong, Y. K. Functional Group Interactions of Adsorbed Small Charged Bolaform Molecules and Their Effects on Intermolecular and Surface Forces in Dispersions. Colloids and Surfaces, $a$ Physicochemical and Engineering Aspects 2008, 325 (3), 127-131.

8. Leong, Y. K. Role of Molecular Architecture of Citric and Related Polyacids on the Yield Stress of alpha-Alumina Slurries: Inter- and Intramolecular Forces. J. Am. Ceram. Soc. 2010, 93 (9), 25982605.

9. Leong, Y. K. Particle Bridging in Dispersions by Small Charged Molecules: Chain Length and Rigidity, Architecture and Functional Groups Spatial Position. Phys. Chem. Chem. Phys. 2007, 9, 56085618.

10. Biggs, S. Steric and Bridging Forces between Surfaces Bearing Adsorbed Polymer - an AtomicForce-Microscopy Study. Langmuir 1995, 11 (1), 156-162.

11. Pedersen, H. G.; Bergstrom, L. Forces Measured between Zirconia Surfaces in Poly(acrylic acid) Solutions. J. Am. Ceram. Soc. 1999, 82 (5), 1137-1145.

12. Notley, S. M.; Leong, Y.-K. Interaction between Silica in the Presence of Adsorbed Poly(Ethyleneimine): Correlation between Colloidal Probe Adhesion Measurements and Yield Stress. Phys. Chem. Chem. Phys. 12 (35), 10594-10601.

13. Parsons, D. F.; Walsh, R. B.; Craig, V. S. J. Surface forces: Surface roughness in theory and experiment. The Journal of Chemical Physics 2014, 140 (16), 164701.

14. Walsh, R. B.; Nelson, A.; Skinner, W. M.; Parsons, D.; Craig, V. S. J. Direct Measurement of van der Waals and Diffuse Double-Layer Forces between Titanium Dioxide Surfaces Produced by Atomic Layer Deposition. The Journal of Physical Chemistry C 2012, 116 (14), 7838-7847.

15. Ducker, W. A.; Senden, T. J.; Pashley, R. M. Direct Measurement of Colloidal Forces using an Atomic Force Microscope. Nature 1991, 353 (6341), 239-241. 
16. Butt, H. J. Measuring Electrostatic, van der Waals and Hydration Forces in ElectrolyteSolutions with an Atomic Force Microscope. Biophys. J. 1991, 60 (6), 1438-1444.

17. Dijt, J. C.; Stuart, M. A. C.; Hofman, J. E.; Fleer, G. J. Kinetics of Polymer Adsorption in Stagnation Point Flow. Colloids Surf. 1990, 51, 141-158.

18. Howard, S. C.; Craig, V. S. J. Very Slow Surfactant Adsorption at the Solid-Liquid Interface Is Due to Long Lived Surface Aggregates. Soft Matter 2009, 5 (16), 3061-3069.

19. Dzuy, N. Q.; Boger, D. V. Yield Stress Measurement for Concentrated Suspensions. Journal of Rheology 1983, 27 (4), 321-349.

20. Kosmulski, M.; Matijevic, E. Zeta Potential of Anatase (TiO2) in Mixed Solvents. Colloids Surf. 1992, 64 (1), 57-65.

21. Wiese, G. R.; Healy, T. W. Solubility Effects in Al2O3 and TiO2 Colloidal Dispersions. J. Colloid Interface Sci. 1975, 52 (3), 452-457.

22. Guo, J.; Tiu, C.; Uhlherr, P. H. T.; Fang, T. N. Yielding Behaviour of Organically Treated Anatase TiO2 Suspension. Korea-Australia Rheology Journal 2003, 15 (1), 9-17.

23. Rao, A. S. Electrophoretic Mobility of Alumina, Titania and Their Mixtures in Aqueous Dispersions. Ceram. Int. 1988, 14 (2), 71-76.

24. Wilhelm, P.; Stephan, D. On-Line Tracking of the Coating of Nanoscaled Silica with Titania Nanoparticles via Zeta-Potential Measurements. J. Colloid Interface Sci. 2006, 293 (1), 88-92.

25. Parks, G. A. Isoelectric Points of Solid Oxides Solid Hydroxides and Aqueous Hydroxo Complex Systems. Chem. Rev. 1965, 65 (2), 177-\&.

26. Kosmulski, M. pH-Dependent Surface Charging and Points of Zero Charge - II. Update. J. Colloid Interface Sci. 2004, 275 (1), 214-224.

27. Kosmulski, M. pH-Dependent Surface Charging and Points of Zero Charge III. Update. J. Colloid Interface Sci. 2006, 298 (2), 730-741.

28. Larson, I.; Drummond, C. J.; Chan, D. Y. C.; Grieser, F. Direct Force Measurements Between TiO2 Surfaces. J. Am. Chem. Soc. 1993, 115 (25), 11885-11890.

29. Verwey, E. J. W.; Overbeek, J. T. G. Theory of the Stability of Lyophobic Colloids; Elsevier: Amsterdam, The Netherlands, 1948.

30. Rodríguez, R.; Blesa, M. A.; Regazzoni, A. E. Surface Complexation at the TiO2(anatase)/Aqueous Solution Interface: Chemisorption of Catechol. J. Colloid Interface Sci. 1996, $177(1), 122-131$.

31. Hwang, Y. S.; Lenhart, J. J. Adsorption of C4-Dicarboxylic Acids at the Hematite/Water Interface. Langmuir 2008, 24 (24), 13934-13943.

32. Dobson, K. D.; McQuillan, A. J. In Situ Infrared Spectroscopic Analysis of the Adsorption of Aliphatic Carboxylic Acids to TiO2, ZrO2, Al2O3, and Ta2O5 from Aqueous Solutions. Spectrochimica Acta Part a-Molecular and Biomolecular Spectroscopy 1999, 55 (7-8), 1395-1405.

33. Hwang, Y. S. Adsorption Of Naturally-Occurring Dicarboxylic Acids At The Hematite/Water Interface. Master's Thesis, The Ohio State University2008.

34. Yao, H. L.; Yeh, H. H. Fumarate, Maleate and Succinate Adsorption on Hydrous Delta-Al2O3 2. Electrophoresis Observations and lonic-Strength Effects on Adsorption. Langmuir 1996, 12 (12), 29892994.

35. Johnson, S. B.; Brown, G. E.; Healy, T. W.; Scales, P. J. Adsorption of Organic Matter at Mineral/Water Interfaces. 6. Effect of Inner-Sphere versus Outer-Sphere Adsorption on Colloidal Stability. Langmuir 2005, 21 (14), 6356-6365.

36. Leong, Y. K. Yield Stress and Zeta Potential of Nanoparticulate Silica Dispersions under the Influence of Adsorbed Hydrolysis Products of Metal Ions - Cu(II), AI(III) and Th(IV). J. Colloid Interface Sci. 2005, 292 (2), 557-566.

37. Ong, B. C.; Leong, Y. K.; Chen, S. B. Interparticle Forces in Spherical Monodispersed Silica Dispersions: Effects of Branched Polyethylenimine and Molecular Weight. J. Colloid Interface Sci. 2009, 337 (1), 24-31. 
38. Kosmulski, M. The pH-Dependent Surface Charging and the Points of Zero Charge. J. Colloid Interface Sci. 2002, 253 (1), 77-87.

39. Walsh, R. B. Growing Ultrasmooth Metal Oxide Surfaces for Atomic Force Microscopy Measurements via Atomic Layer Deposition. 2011.

40. Biggs, S.; Scales, P. J.; Leong, Y.-K.; Healy, T. W. Effects of Citrate Adsorption on the Interactions between Zirconia Surfaces. J. Chem. Soc., Faraday Trans. 1995, 91 (17), 2921-2928.

41. Teh, E. J.; Leong, Y. K.; Liu, Y.; Fourie, A. B.; Fahey, M. Differences in the Rheology and Surface Chemistry of Kaolin Clay Slurries: The Source of the Variations. Chem. Eng. Sci. 2009, 64 (17), 38173825 .

42. Leong, Y. K. Interparticle Forces Arising from an Adsorbed Strong Polyelectrolyte in Colloidal Dispersions: Charged Patch Attraction. Colloid Polym. Sci. 1999, 277 (4), 299-305.

43. Kosmulski, M.; Rosenholm, J. B. Electroacoustic Study of Adsorption of Ions on Anatase and Zirconia from Very Concentrated Electrolytes. J. Phys. Chem. 1996, 100 (28), 11681-11687.

44. Khoo, K. S.; Teh, E. J.; Leong, Y. K.; Ong, B. C. Hydrogen Bonding and Interparticle Forces in Platelet alpha-AI2O3 Dispersions: Yield Stress and Zeta Potential. Langmuir 2009, 25 (6), 3418-3424.

45. Leong, Y. K.; Scales, P. J.; Healy, T. W.; Boger, D. V.; Buscall, R. Rheological Evidence of Adsorbate-Mediated Short-range Steric Forces in Concentrated Dispersions. J. Chem. Soc., Faraday Trans. 1993, 89 (14), 2473-2478.

46. Hwang, Y. S.; Lenhart, J. J. Dicarboxylic Acid Transport through Hematite-Coated Sand. Chemosphere 78 (8), 1049-1055.

47. Johnson, S. B.; Franks, G. V.; Scales, P. J.; Boger, D. V.; Healy, T. W. Surface Chemistry-Rheology Relationships in Concentrated Mineral Suspensions. Int. J. Miner. Process. 2000, 58 (1-4), 267-304.

48. Teh, E. J.; Leong, Y.-K.; Liu, Y. The Effect of Adsorbed Fumaric Acid on Dispersions of Rough Titania Particles. Powder Technol. 223, 110-115. 